\title{
1 ISWFoam: A numerical model for internal solitary wave 2 simulation in continuously stratified fluids
}

3 Jingyuan $\mathrm{Li}^{1}$, Qinghe Zhang ${ }^{1}$, Tongqing $\mathrm{Chen}^{1}$

$4{ }^{1}$ State Key Laboratory of Hydraulic Engineering Simulation and Safety, Tianjin University, Tianjin 5300072 , China

6 Correspondence to: Qinghe Zhang (qhzhang@tju.edu.cn)

7 Abstract. A numerical model, ISWFoam, for simulating internal solitary waves (ISWs) in continuously 8 stratified, incompressible, viscous fluids is developed based on a fully three-dimensional (3D) Navier-

9 Stokes equation using the open source code OpenFOAM. This model combines the density transport equation with the Reynolds-averaged Navier-Stokes equation with the Coriolis force, and the model discrete equation adopts the finite volume method. The $k-\omega S S T$ turbulence model has also been modified accordingly to the variable density field. ISWFoam provides two initial wave generation methods to generate an ISW in continuously stratified fluids, including solving the weakly nonlinear models of the extended Korteweg-de Vries (eKdV) equation and the fully nonlinear models of the Dubreil-JacotinLong (DJL) equation. Grid independence tests for ISWFoam are performed, considering the accuracy and computing efficiency, the appropriate grid size of the ISW simulation is recommended to be oneone hundred and fiftieth of the characteristic length and one-twenty fifth of the ISW amplitude. Model verifications are conducted through comparisons between the simulated and experimental data for ISW propagation examples over a flat bottom section, including laboratory scale and actual ocean scale, a submerged triangular ridge, a Gaussian ridge and slope. The laboratory test results, including the ISW profile, wave breaking location, ISW arrival time, and the spatial and temporal changes in the mixture region, are well reproduced by ISWFoam. The ISWFoam model with unstructured grids and local mesh refinement can accurately simulate the generation and evolution of ISWs, the ISW breaking phenomenon and the interaction between ISWs and complex structures and topography.

Key words. OpenFoam, Internal solitary wave tank, Stratified fluid, the DJL equation, Grid independence.

\section{Introduction}

Internal solitary waves (ISWs) are commonly observed in oceans, particularly on continental shelf 
regions, due to strong tidal current flows over large topographic features (Huthnance, 1981), such as in the northern South China Sea (Alford et al., 2010; Alford et al., 2015; Cai et al., 2012). ISWs play an important role in both conveying nutrients from the deep ocean to shallower layers and promoting biological growth (Sandstrom et al., 1984). Additionally, ISWs are a potential threat to the ocean structures of resource exploration, exploitation, and submarine navigation vehicles (Alford et al., 2010; Osborne et al., 1980). A considerable number of studies, which include field measurements, remote sensing, experiments, theoretical analysis and numerical simulations, have been carried out due to the significance of ISWs (Vlasenko et al., 2005; Apel et al., 2006; Alford et al., 2011; Guo et al., 2014).

For numerically simulated ISWs, many models have been adopted, including the Euler equation, the inviscid/viscid incompressible Boussinesq model, the hydrostatic model, the non-hydrostatic model, and the VOF based two-phase flow model. Among these models, the representative hydrostatic models include the Naval Research Laboratory Ocean Nowcast/Forecast System (ONFS) (Ko et al.,2008), the Regional Hallberg Isopycnal Tide Mode (RHIMT) (Hallberg and Rhines, 1996; Hallberg, 1997), and the Ostrovsky-Hunter model. The representative non-hydrostatic models include the Bergen Ocean Model (BOM), the nonhydrostatic Regional Ocean Modeling System model (ROMS), the Stanford Unstructured Nonhydrostatic Terrain-following Adaptive Navier-Stokes Simulator (SUNTANS), and the Massachusetts Institute of Technology general circulation model (MITgcm). For example, Zhang et al (2012) established a variable water depth internal wave numerical model in a continuously stratified fluid system based on the Euler equation. Xu and Stastna (2020) used the viscid incompressible Boussinesq model to study cross-boundary-layer transport (Boegman and Stastna, 2019) by the fissioning process of shoaling ISWs. Lamb (1994) established a non-hydrostatic model, using a second-order projection method developed by Bell and Marcus (1992), which is used for internal wave research including boundary layer instability (Aghsaee et al., 2012), reflection (Lamb, 2009), and the interaction of the tides with the topography (Lamb, 2007; Aghsaee et al., 2010). Diamessis (2005) developed a spectral multidomain penalty method model and correctly reproduced the characteristic vorticity and internal wave structure. Subich et al (2013) developed a spectral collocation method for the solution of the Navier-Stokes equations under the Boussinesq approximation, and simulated the internal wave in continuously stratified fluid. Smedstad et al (2003) employed the ONFS model to establish a global ocean real-time forecasting system with an operational eddy resolution of $1 / 16^{\circ}$, which effectively tracks ocean 
https://doi.org/10.5194/gmd-2021-102

Preprint. Discussion started: 27 May 2021

(c) Author(s) 2021. CC BY 4.0 License.

eddies, ocean currents and ocean fronts. Simmons et al (2004) employed the RHIMT model to carry out a global numerical simulation of tidal currents, and analyzed the whole process of the conversion rate of barotropic waves into baroclinic waves. Thiem (2011) used the Bergen Ocean Model to explore the bottom boundary layer flow caused by waves beneath a propagating ISW in a two-fluid system. Li and Farmer (2011) employed the Ostrovsky-Hunter model to study the nonlinear evolution of a monochromatic internal wave. Buijsman et al (2010) employed ROMS model to study the asymmetry in solitons to the east and west of Luzon Strait. Zhang et al (2011) used the nonhydrostatic SUNTANS model (Fringer et al., 2006) to study the dynamics of A wave and B wave formation. Rayson et al (2018) used the modified SUNTANS model to study the internal waves around Scott Reef and provided the generation process of internal lee waves. Vlasenko et al (2010) employed the MITgcm model to investigate the baroclinic tidal energy conversion in the area west of the Luzon Strait.

In summary, for continuously stratified fluids in complex ocean environments, numerical simulation has become a leading method for ISW investigations. However, there are presently few versatile numerical models with share code that can accurately simulate the ISW flow around complex topography and submarine navigation vehicles in continuously stratified fluids. Therefore, the main objective of this paper is to develop a solver, referred to as ISWFoam with a modified $k$ - $\omega S S T$ model that considers the variable density field, which simulates the ISW in continuous density stratification, incompressible and viscous fluids using the finite volume method with unstructured grids based on a fully three-dimensional (3D) Navier-Stokes equation using the OpenFOAM library.

Notably, the open source field operation and manipulation code OpenFOAM ${ }$, as an objectoriented $\mathrm{C}++$ open source library that can be used to build a variety of solvers for computational fluid problems based on the finite volume method, is becoming increasingly popular in the computational fluid research community. At present, the official version of OpenFOAM ${ }^{\circledR}$ does not have a solver or boundary conditions for solving the ISW in continuously stratified fluids. Although some researchers simulate ISWs by modifying the OpenFOAM ${ }^{\circledR}$ code, most of these studies are based on a two-fluid system without considering continuous stratification in density, such as Meng and Zhang (2016) and Li et al (2017). Though recent work by Ding et al (2020) and Li et al (2021) considered continuous stratification in density, the wave generation method is essential for a two-layer system. To extensively use of the numerical model of ISWs as a tool in the future, we will develop ISWFoam to simulate the ISW in 
continuously stratified, incompressible and viscous fluids based on the OpenFOAM library. The turbulence model will consider the variable density field. In addition, ISWFoam will provide two initial methods to generate an ISW in continuously stratified fluids, including solving the weakly nonlinear models of the extended Korteweg-de Vries (eKdV) equation and the fully nonlinear models of the Dubreil-Jacotin-Long (DJL) equation. This approach renders the numerical model suitable for the simulation of ISW flows in complex geometries and topographies.

The outline of the paper is described as follows. First, in Section 2, the governing equations for a continuously stratified fluid are presented, and discrete forms of these equations are derived. Then, grid independence tests of the developed ISWFoam model are described in Section 3. Subsequently, in Section 4, a series of test cases are presented to verify the model. Finally, the conclusions are drawn in Section 5.

\section{ISWFoam: A three-dimensional numerical solver for ISWs in a continuously stratified fluid}

\subsection{Governing equations}

We present an ISW numerical model by solving the motion of a three-dimensional, viscous, incompressible fluid with the Boussinesq approximation and rigid lid hypothesis. The governing equations of the model are

$$
\nabla \cdot \mathbf{U}=0
$$

$$
\frac{\partial \mathbf{U}}{\partial t}+(\mathbf{U} \cdot \nabla) \mathbf{U}-\nabla \cdot\left(v_{E f f} \nabla \mathbf{U}\right)=\mathbf{Q} \quad \mathbf{Q}=\frac{1}{\rho_{0}}\left(-\nabla p_{-r g h}-\mathbf{g} \cdot \mathbf{X} \nabla \rho-\Omega e_{3}\right),
$$

$$
\frac{\partial \rho}{\partial t}+(\mathbf{U} \cdot \nabla) \rho=\nabla \cdot(k \nabla \rho)
$$

where $\mathbf{U}=\left(u_{i}, u_{j}, u_{k}\right)$ is the velocity vector, $t$ is time, $\nabla$ is the gradient operator, $\mathbf{Q}$ is the source term, $\rho_{0}$ is the reference density, $\rho$ is the density field, $p_{-r g h}$ is a modified pressure field, $\mathbf{g}$ is the gravitational acceleration vector, and $\mathbf{X}$ is the position vector. $v_{E f f}$ is the effective kinematic viscosity defined as $v_{E f f}=$ $\mu_{E f f} / \rho_{0}$, where $\mu_{E f f}$ is the effective dynamic viscosity including the molecular viscosity $\left(\mu_{l}\right)$ and turbulent $\operatorname{viscosity}\left(\mu_{t}\right) . k$ is the diffusion coefficient, and its value is the same as the effective dynamic $\operatorname{viscosity}\left(\mu_{E f f}\right) . \Omega$ is the Coriolis parameter, which is the twice the speed of rotation around the vertical unit vector $e_{3}=(0,0,1)$. ISWFoam uses a modified pressure $p_{-}$rgh instead of a total pressure $p$, and their relationship is given by 


$$
p_{-\mathrm{rgh}}=p-\rho \mathbf{g} \cdot \mathbf{X}, \nabla p_{\mathrm{r}_{\mathrm{rgh}}}=\nabla p-\rho \mathbf{g}-\mathbf{g} \cdot \mathbf{X} \nabla \rho,
$$

To close the above equations, the turbulence model needs to be employed. The two-equation $k-\varepsilon$ model is widely used as an effective turbulence model, but it cannot capture the proper behaviour of turbulent boundary layers up to separation due to adverse pressure gradients (Wilcox, 1993). For the above boundary layers separation problem, Bardina et al. (1997) and Menter et al. (2003) suggested the use of the $k$ - $\omega S S T$ model to obtain substantially more accurate results. Therefore, the turbulence model used in this paper is the $k$ - $\omega S S T$ model. Notably that in OpenFOAM, the incompressible version for turbulence models does not consider the variable density field, and instead, it treats the density as a constant, such as the $k-\omega S S T$ model

$$
\frac{\partial k}{\partial t}+\nabla \cdot(\mathbf{U} k)=\nabla \cdot\left[\left(v_{E f f}+\sigma_{k} v_{t}\right) \nabla k\right]+P_{k}^{*}-\beta^{*} \omega k
$$

$$
\frac{\partial \omega}{\partial t}+\nabla \cdot(\mathbf{U} \omega)=\nabla \cdot\left[\left(v_{E f f}+\sigma_{\omega} v_{t}\right) \nabla \omega\right]+C_{\gamma} \frac{\omega}{k} P_{k}-C_{\beta} \omega^{2}+2\left(1-F_{1}\right) \frac{\sigma_{\omega 2}}{\omega} \nabla k \cdot \nabla \omega
$$

where $k$ is the turbulent kinetic energy, $\omega$ is the specific dissipation rate, $P_{k}$ is the production term of $k$, $P_{k}=\tau^{R}: \nabla \mathbf{U}, P_{k}^{*}$ is related to the production term of turbulence kinetic energy $P_{k}$ in the $k$ equation, $v_{t}$ is the turbulent kinematic viscosity, $S_{t}$ is the mean rate of the flow strain, $S_{t}=0.5\left(\nabla \mathbf{U}+\nabla \mathbf{U}^{T}\right)$, the model constants are assigned the values $\beta^{*}=0.09, a_{1}=0.31, c_{1}=10$ and $C_{\mu}=0.09, F_{1}$ and $F_{2}$ are blending functions, the value of $\sigma_{k}, \sigma_{\omega}, C_{\gamma}$ and $C_{\beta}$ are blended using the equation $\Phi=F_{1} \Phi_{1}+\left(1-F_{1}\right) \Phi_{2}$ in which $\Phi_{1}$ and $\Phi_{2}$ are given in Table 1.

Table 1 Default values for $\Phi_{1}$ and $\Phi_{2}$

\begin{tabular}{ccccc}
\hline$\Phi$ & $\sigma_{k}$ & $\sigma_{\omega}$ & $C_{\beta}$ & $C_{\gamma}$ \\
\hline$\Phi_{1}$ & 0.85 & 0.5 & 0.075 & $5 / 9$ \\
$\Phi_{2}$ & 1.0 & 0.856 & 0.0828 & 0.44 \\
\hline
\end{tabular}

Considering the variable density field during the solution process, it is necessary to consider the change in the density field in the turbulence model. Therefore, we modify the turbulence model to consider the change in density, and finally a modified $k$ - $\omega S S T$ model that considers the change in density is used to close the equation 


$$
\frac{\partial \rho \omega}{\partial t}+\nabla \cdot(\rho \mathbf{U} \omega)=\nabla \cdot\left[\rho\left(v_{E f f}+\sigma_{\omega} v_{t}\right) \nabla \omega\right]+C_{\gamma} \frac{\omega}{k} P_{k}-C_{\beta} \rho \omega^{2}+2\left(1-F_{1}\right) \rho \frac{\sigma_{\omega 2}}{\omega} \nabla k \cdot \nabla \omega
$$

\subsection{Numerical discretization} $\mathrm{C}++$ open source library of OpenFOAM. The finite volume method requires that Eqs. (2) and (3) are satisfied over the control volume $V_{\mathrm{P}}$ around point $\mathrm{P}$ in integral form: defined to represent the current moment. The PISO iteration process is marked as $m$; when $m$ is equal to zero, it represents the initial moment $\left(t^{\mathrm{n}}\right)$. expression for the predicted velocity field $\mathbf{U}_{P}^{r}$, namely,

$$
\frac{\mathbf{U}_{P}^{r}-\mathbf{U}_{P}^{n}}{\Delta t} V_{P}+\sum_{f \in \partial V_{P}}\left(\phi_{f}^{n} \mathbf{U}_{f}^{r}\right)-\sum_{f \in \partial V_{P}} v_{E f f} \nabla \mathbf{U}_{f}^{r} \cdot \mathbf{S}_{f}=0
$$
$\mathbf{S}_{f}$ is the face vector. the centre $P$ of the grid and the centre $N$ of the adjacent grid, the face values are calculated using a mixture method (blended differencing) of the central scheme (central differencing) and the upwind scheme (upwind differencing) as follows (Jasak, 1996):

$$
\mathbf{U}_{f}=\left(1-\lambda_{U}\right)\left(\mathbf{U}_{f}\right)_{U D}+\lambda_{U}\left(\mathbf{U}_{f}\right)_{C D}
$$

where 


$$
\left(\mathbf{U}_{f}\right)_{U D}=\left\{\begin{array}{l}
\mathbf{U}_{P} \text { for } \phi_{f} \geq 0, \\
\mathbf{U}_{N} \text { for } \phi_{f}<0,
\end{array} \quad \text { and }\left(\mathbf{U}_{f}\right)_{C D}=\frac{\mathbf{U}_{P}+\mathbf{U}_{N}}{2}\right.
$$

$$
\mathbf{U}_{f}=\frac{1}{2}\left(\mathbf{U}_{P}+\mathbf{U}_{N}\right)+\frac{1}{2}\left[\psi\left(\phi_{f}\right)\left(1-\lambda_{U}\right)\right]\left(\mathbf{U}_{P}-\mathbf{U}_{N}\right),
$$

where $\psi\left(\phi_{f}\right)$ is a step function defined by

$$
\psi\left(\phi_{f}\right)=\left\{\begin{array}{c}
1 \text { for } \phi_{f} \geq 0, \\
-1 \text { for } \phi_{f}<0,
\end{array}\right.
$$

$$
A_{P} \mathbf{U}_{P}^{r}=\sum_{f \in \partial V_{\mathrm{P}}} A_{N} \mathbf{U}_{P}^{m}+\frac{\mathbf{U}_{P}^{n}}{\Delta t}=H\left(\mathbf{U}^{m}\right)
$$

After some manipulation, the quantities $A_{P}$ and $A_{N}$ are given as

$$
\begin{aligned}
& A_{P}=\left\{\frac{V_{\mathrm{P}}}{\Delta t}+\sum_{f \in \partial V_{\mathrm{P}}} \frac{\phi_{f}^{n}}{2}\left[1+\psi\left(\phi_{f}\right)\left(1-\lambda_{U}\right)\right]+\sum_{f \in \partial V_{\mathrm{P}}} v_{E f f, f} \frac{\left|\mathbf{S}_{f}\right|}{|d|}\right\} \frac{1}{V_{\mathrm{P}}} \\
& A_{N}=\left\{-\frac{\phi_{f}^{n}}{2}\left[1-\psi\left(\phi_{f}\right)\left(1-\lambda_{U}\right)\right]+v_{E f f, f} \frac{\left|\mathbf{S}_{f}\right|}{|d|}\right\} \frac{1}{V_{\mathrm{P}}}
\end{aligned}
$$

$$
\mathbf{U}_{P}^{r}=\frac{H\left(\mathbf{U}^{m}\right)}{A_{P}}-\frac{\left(\mathbf{g} \cdot \mathrm{X} \nabla \rho / \rho_{0}\right)^{n}}{A_{P}}-\frac{\left(\Omega e_{3}\right)^{n}}{A_{P}},
$$

Notably, that when $m$ is equal to zero, it represents the initial moment $n$, and the value of the initial moment is known. Therefore, we obtain the predicted velocity field $\mathbf{U}_{P}^{r}$ in the first iteration. We define the surface gradient operator $\left(\nabla \frac{1}{f}\right)$, and the type of gradient operator acting on $\mathbf{U}$ is $\nabla \frac{1}{f} \mathbf{U}=\left(\mathbf{U}_{N}^{m}-\mathbf{U}_{P}^{m}\right) /|d|$, which represents the distance from the centre of the grid $N$ to $P$. Similarly, the surface gradient operator $\left(\nabla \frac{1}{f}\right)$ acting on scalar $\gamma$ is $\nabla \frac{1}{f} \lambda=\left(\lambda_{N}^{m}-\lambda_{P}^{m}\right) /|d|$. The associated flux $\left(\phi_{f}=\mathbf{U}_{f} \cdot \mathbf{S}_{f}\right)$ is achieved by executing an inner product with a surface vector $\left(\mathbf{S}_{f}\right)$ on the left and right 


$$
\phi_{f}^{r}=\left(\frac{H\left(\mathbf{U}^{m}\right)}{A_{P}}\right)_{f} \cdot \mathbf{S}_{f}-\left(\left(\frac{1}{A_{P}}\right)_{f}(\mathbf{g} \cdot \mathbf{X})_{f}^{n}\left(\frac{1}{\rho_{0}} \nabla \frac{1}{f} \rho\right)^{n}\left|\mathbf{S}_{f}\right|\right)-\left(\frac{\left(\Omega e_{3}\right)^{n}}{A_{P}}\right)_{f} \cdot \mathbf{S}_{f},
$$

186

Eq. (22) completed the flux calculation without considering the influence of the pressure term. The pressure contribution in terms of a flux can be expressed as

$$
\left(\frac{-\nabla p_{-r g h}}{\rho_{0} A_{P}}\right)_{f} \cdot \mathbf{S}_{f}=\left(\frac{-1}{A_{P}}\right)_{f}\left(\frac{1}{\rho_{0}} \nabla \frac{1}{f} p_{-r g h}^{m+1}\right)\left|\mathbf{S}_{f}\right|,
$$

Then, Eq. (23) is now added to Eq. (22) to yield

$$
\phi_{f}^{m+1}=\left(\frac{H\left(\mathbf{U}^{m}\right)}{A_{P}}\right)_{f} \cdot \mathbf{S}_{f}-\left(\left(\frac{1}{A_{P}}\right)_{f}(\mathbf{g} \cdot \mathbf{X})_{f}^{n}\left(\frac{1}{\rho_{0}} \nabla \frac{1}{f} \rho\right)^{n}\left|\mathbf{S}_{f}\right|\right)-\left(\frac{\left(\Omega e_{3}\right)^{n}}{A_{P}}\right)_{f} \cdot \mathbf{S}_{f}-\left(\frac{1}{A_{P}}\right)_{f}\left(\frac{1}{\rho_{0}} \nabla \frac{1}{f} p_{r g h}^{m+1}\right)\left|\mathbf{S}_{f}\right|
$$

Combined with Eq. (22), Eq. (24) is simplified and rewritten as

$$
\phi_{f}^{m+1}=\phi_{f}^{r}-\left(\frac{1}{A_{P}}\right)_{f}\left(\frac{1}{\rho_{0}} \nabla \frac{1}{f} p_{-r g h}^{m+1}\right)\left|\mathbf{S}_{f}\right|
$$

Using conservation of mass, we solve the pressure field $p_{-r g h}^{m+1}$, which results in

$$
\sum_{f \in \partial V_{\mathrm{P}}}\left(\frac{1}{A_{P}}\right)_{f}\left(\frac{1}{\rho_{0}} \nabla \frac{1}{f} p_{-r g h}^{m+1}\right)\left|\mathbf{S}_{f}\right|=\sum_{f \in \partial V_{\mathrm{P}}} \phi_{f}^{r}
$$

$$
\text { The preconditioned conjugate gradient method is used to solve the linear system constructed by Eq. }
$$

(26) (OpenFOAM, 2019). After $p_{-r g h}^{m+1}$ is obtained using Eq. (26), we calculate the volume flux using

Eq. (25) for each face. The cell centred velocity fields $\mathbf{U}_{P}^{m+1}$ are calculated by reconstructing the face velocity flux using the following expression (Deshpande, 2012)

$$
\mathbf{U}_{P}^{m+1}=\mathbf{U}_{P}^{r}+\left(\frac{1}{A_{P}}\right)\left(\sum_{f \in \partial V_{\mathrm{P}}} \frac{\mathbf{S}_{f} \mathbf{S}_{f}}{\left|\mathbf{S}_{f}\right|}\right)^{-1} \cdot\left(\sum_{f \in \partial V_{\mathrm{P}}}\left(\frac{\phi_{f}^{m+1}-\left(\mathbf{U}_{P}^{r}\right)_{f} \cdot \mathbf{S}_{f}}{\left(1 / A_{P}\right)_{f}}\right) \frac{\mathbf{S}_{f}}{\left|\mathbf{S}_{f}\right|}\right)
$$

Eq. (27) completes the velocity field calculation of the first iteration step in the PISO algorithm. By converting the identifier $m$ to $m+1$, the next PISO iteration is completed and updating the velocity in Eq. (18) with the velocity $\mathbf{U}_{P}^{m+1}$ calculated from Eq. (27), thereby updating $p_{-} r g h, \phi_{f}$ and $\mathbf{U}$. This procedure is performed $M$ times to guarantee that the results of the velocity and pressure together conform to the continuity and momentum equations. Considering that PISO iteration levels require more than 1 , but typically not more than 4 (OpenFOAM, 2019), we specify that the number of PISO iteration levels is 3 
in the computations presented in this paper. After completing the three iterations, the converged values are considered the result of the next time step $(n+1)$, namely,

$$
\phi_{f}^{n+1}=\phi_{f}^{M}, \quad \mathbf{U}_{P}^{n+1}=\mathbf{U}_{P}^{M}, \quad p_{-r g h}^{n+1}=p_{-r g h}^{M},
$$

We discretize the convection-diffusion equation of density (12) to obtain

$$
\frac{V_{P}}{\Delta t}\left(\rho_{P}^{n+1}-\rho_{P}^{n}\right)+\sum\left(\phi_{f}^{n+1} \rho_{f}^{n+1}\right)=\sum k\left[\left|\mathbf{S}_{f}\right| \frac{\rho_{N}^{n+1}-\rho_{P}^{n+1}}{|d|}\right],
$$

At the end of the iteration procedure, we bring the results of the volume flux into Eq. (29) to calculate the density field at the next time $\left(\rho_{\mathrm{P}}^{n+1}\right)$, thereby updating the density field for the next step calculation $\left(\Delta t=t^{n+2}-t^{n+1}\right)$.

\subsection{Initialized field of ISW generation}

ISW generation methods mainly include the gravity collapse mechanism, double push-pedals method (Fu et al., 2008), velocity-inlet method (Gao et al., 2012), mass source method (Wang et al., 2018), initialization method, and methods addressing the interaction between tidal current and topography. For example, Hsieh et al (2014) investigated the flow evolution of a depression ISW generated by the gravity collapse mechanism. Cheng et al (2020) studied the interaction between ISWs and a cylinder using the gravity collapse mechanism. The initialization method involves solving the internal solitary wave theory at the initial moment, such as the Korteweg-de Vries (KdV) equation (Grimshaw et al., 2010), the modified $\mathrm{KdV}(\mathrm{mKdV})$ equation, the extended $\mathrm{KdV}$ (eKdV) equation, the forced KdV equation, the Ostrovsky equation (Li and Farmer, 2011), the Miyata-Choi-Camassa (MCC) model (Miyata 1985 and 1988; Choi and Camassa, 1999), and the Dubreil-Jacotin-Long (DJL) equation (Long, 1953; Turkington, 1991; Brown and Christie, 1997; Dunphy et al., 2011), to obtain the wave surface, velocity field. The method of an interacting between tidal current and terrain that stimulates ISWs is adopted by many scholars, such as Farmer and Smith (1980), Lamb et al (1994), and Shaw et al (2009).

In this paper, the method of initializing the field is selected to generate the ISWs. To increase the application range of the ISWFoam model, two initialization methods are provided, including solving the weakly nonlinear models of the eKdV equation (Helfrich and Melville, 2006) and the fully nonlinear models of the DJL equation for continuously stratified fluids (Turkington, 1991; Dunphy et al, 2011). 
The Dubreil-Jacotin-Long (DJL) equation is expressed as

$$
\begin{aligned}
\nabla^{2} \eta+\frac{N^{2}(z-\eta)}{c^{2}} \eta=0, & \eta=0 \quad \text { at } \quad z=0,-H \\
& \eta=0 \quad \text { at }|x| \rightarrow \infty
\end{aligned}
$$

where $\eta$ is the isopycnal displacement, $H$ is the water depth, $c$ is the propagation speed, $N$ is the definition of the buoyancy frequency, and $z$ is vertical position.

$$
N^{2}(z)=-g \frac{d \rho_{0}(\mathrm{z})}{d z},
$$

where $\rho_{0}(z)$ is the reference density, and $g$ is the gravitational acceleration.

By solving the above DJL equation we can obtain $\eta$ and $c$, and then through the relationship $\psi=\eta c$, where $\psi$ is the stream function, we can obtain the wave-induced velocity field. We use the DJLES open source package provided by Dunphy et al (2011) to solve the DJL equations. Then we input the initial field calculated by DJLES into OpenFOAM to obtain the initial field required for OpenFOAM numerical simulation.

Another theory of ISWFoam model wave generation involves the weakly nonlinear models of the $\mathrm{eKdV}$ equation. Using the first order stream function for the DJL equation, we can obtain the well-known $\mathrm{KdV}$ equation and further obtain the eKdV equation. For the specific derivation, please refer to the paper by Lamb and Yan (1996). The eKdV equation (Helfrich and Melville, 2006) is

$$
\begin{aligned}
& \frac{\partial \zeta}{\partial t}+\left(c_{0}+c_{1} \zeta+c_{3} \zeta^{2}\right) \frac{\partial \zeta}{\partial x}+c_{2} \frac{\partial^{3} \zeta}{\partial x^{3}}=0, \\
c_{0}^{2}= & \frac{g h_{1} h_{2}\left(\rho_{2}-\rho_{1}\right)}{\rho_{1} h_{2}+\rho_{2} h_{1}}, \\
c_{1}= & -\frac{3 c_{0}}{2} \frac{\rho_{1} h_{2}^{2}-\rho_{2} h_{1}^{2}}{\rho_{1} h_{1} h_{2}^{2}+\rho_{2} h_{1}^{2} h_{2}}, \\
c_{2}= & \frac{c_{0}}{6} \frac{\rho_{1} h_{1}^{2} h_{2}+\rho_{2} h_{1} h_{2}^{2}}{\rho_{1} h_{2}+\rho_{2} h_{1}}, \\
c_{3}= & \frac{3 c_{0}}{h_{1}^{2} h_{2}^{2}}\left[\frac{7}{8}\left(\frac{\rho_{1} h_{2}^{2}-\rho_{2} h_{1}^{2}}{\rho_{1} h_{2}+\rho_{2} h_{1}}\right)^{2}-\frac{\rho_{1} h_{2}^{3}+\rho_{2} h_{1}^{3}}{\rho_{1} h_{2}+\rho_{2} h_{1}}\right],
\end{aligned}
$$

where $\zeta$ is the isopycnal vertical displacement; $h_{1}$ and $h_{2}$ are the mean upper and lower layer depths, respectively; $\rho_{1}$ and $\rho_{1}$ are the fluid densities of the upper and lower layers, respectively. The theoretical 


$$
\begin{aligned}
& \zeta=\frac{a}{B+(1-B) \cosh ^{2}\left[\lambda_{\mathrm{eKdV}}\left(x-c_{\mathrm{eKdV}} t\right)\right]}, \\
& \lambda_{\mathrm{eKdV}}^{2}=\frac{a}{12 c_{2}}\left(c_{1}+\frac{1}{2} c_{3} a\right), \\
& c_{\mathrm{eKdV}}=c_{0}+\frac{a}{3}\left(c_{1}+\frac{1}{2} c_{3} a\right), \\
& B=\frac{-a c_{3}}{2 c_{1}+a c_{3}}, \\
& u_{1}=-c_{\mathrm{eKdV}} \frac{\zeta}{h_{1}-\zeta}, u_{2}=c_{\mathrm{eKdV}} \frac{\zeta}{h_{2}+\zeta},
\end{aligned}
$$

where $a$ is the ISW amplitude, $\lambda_{\mathrm{eKdV}}$ is the wavelength, $c_{\mathrm{eKdV}}$ is the wave speed, $B$ is an auxiliary parameter, and $u_{1}$ and $u_{2}$ are the speeds of the upper and lower layers of the fluid, respectively. The waveform and velocity field of the ISWs are solved at the initial moment by the developed function and then assigned to the calculation domain.

The vertical profile of the initial density is given by a hyperbolic tangent function profile (Aghsaee et al., 2010)

$$
\bar{\rho}(z)=\frac{\rho_{1}+\rho_{2}}{2}-\frac{\rho_{2}-\rho_{1}}{2} \tanh \left(\frac{z-z_{p y c}}{d_{p y c}}\right)
$$

where $z$ is the vertical position; $\rho_{1}$ and $\rho_{2}$ are the fluid densities of the upper and lower layers, respectively; $z_{p y c}$ is the location of the centre of the pycnocline; and $d_{p y c}$ is the thickness of the pycnocline. In this paper, unless otherwise specified, the form of the density profile adopts Eq. (42). The internal solitary wave surface is obtained by calculating the gradient of the density field, and the absolute value of the maximum value of the gradient represents the vertical position of the wave surface. Notably, the density profile of the actual ocean is not always hyperbolic, so our model provides a function for users to modify the density profile according to the actual situation.

\subsubsection{Comparison between the DJL equation and the eKdV equation}

To compare the DJL equation and the $\mathrm{eKdV}$ equation, we set up a numerical simulation, which includes a tank that is $15 \mathrm{~m}$ long, $1 \mathrm{~m}$ wide and has a water depth of $0.5 \mathrm{~m}$. The depths of the upper $\left(h_{1}\right)$ and lower $\left(h_{2}\right)$ layers are $0.1 \mathrm{~m}$ and $0.4 \mathrm{~m}$, respectively, the densities of the upper and lower layers are $1022 \mathrm{~kg} / \mathrm{m}^{3}$ and $1028 \mathrm{~kg} / \mathrm{m}^{3}$, respectively, the location of the centre of the pycnocline $\left(z_{p y c}\right)$ is $0.4 \mathrm{~m}$, the 
pycnocline thickness $\left(d_{p y c}\right)$ is $0.04 \mathrm{~m}$ vertically, the initial ISW amplitude $(a)$ is $0.065 \mathrm{~m}$ and the location position $10 \mathrm{~m}$ away from the initial ISW. The grid is uniform in the $x$-direction, $y$-direction and $z$-direction, and the sizes are $\Delta x=1 \times 10^{-2} \mathrm{~m}, \Delta y=1 \times 10^{-2} \mathrm{~m}$ and $\Delta z=1 \times 10^{-3} \mathrm{~m}$, respectively. Slip boundary conditions are applied to the bottom and both sides, while cyclic boundary conditions are assigned to the inlet and outlet boundaries. The top boundary is a rigid lid. The boundary conditions related to the density field are no-flux boundary conditions.

Fig. 1 shows the comparison of the horizontal velocity component field when the DJL equation and the $\mathrm{KdV}$ equation are used to generate ISWs. At the initial moment, the ISW generated by the eKdV equation is not as smooth as the ISW generated by the DJL equation, and the horizontal velocity at the interface area is discontinuous as shown in Fig. 1(a) and $(b)$. With the propagation of ISWs, the ISWs generated by the DJL equation are always smooth at the interface area, and the velocity field is always continuous as shown in Fig. 1(a), (c), (e) and $(g)$. Correspondingly, the ISW generated by the eKdV equation gradually produces a gradient in the vertical direction of the horizontal velocity in the interface area, thus, the interface area becomes smooth, and the velocity becomes continuous. Fig. 1(d) shows this evolution process, which is basically completed in 5 s as shown in Fig. 1(f). At 50s, the difference between the horizontal velocity fields of the two equations is very small as shown in Fig. $1(g)$ and $(h)$.
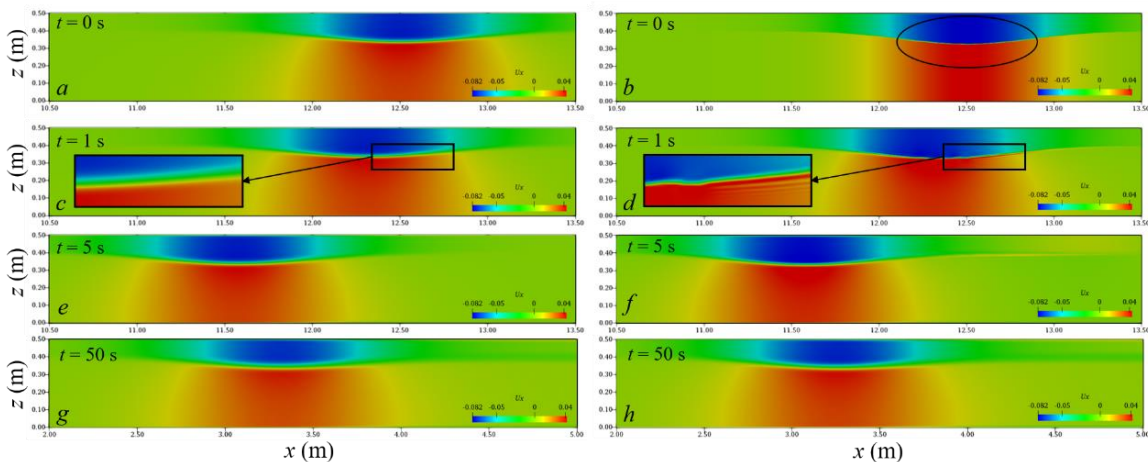

Figure 1: Comparison chart of the horizontal velocity component field: DJL equation (left) and eKdV equation (right).

Fig. 2 shows the comparison of the vertical velocity component field when the DJL equation and

the $\mathrm{eKdV}$ equation are used to generate ISWs. Since the theoretical solution of the eKdV equation only obtain the average horizontal velocity of the upper and lower layers of the fluid, there is no vertical 
field will gradually be generated and finally stabilized, and the stable time occurs at $5 \mathrm{~s}$ as shown in Fig. $2(b),(d),(f)$ and $(h)$. At 50 s, the difference between the vertical velocity fields of the two equations is very small as shown in Fig. $2(g)$ and $(h)$.
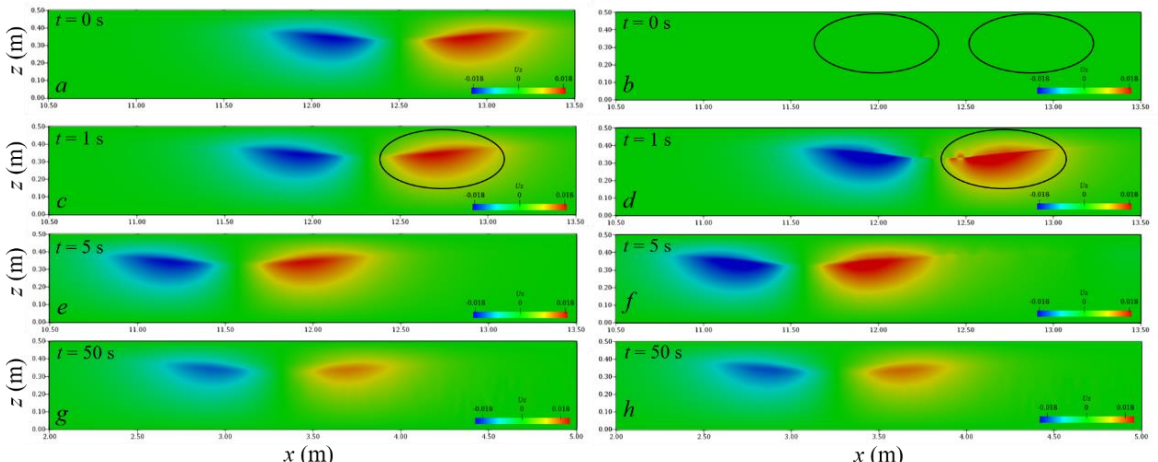

Figuire 2: Comparison chart of the vertical velocity component field: DJL equation (left) and eKdV equation (right).

The ISW propagates for $10 \mathrm{~m}$, and the amplitudes of the ISWs generated by the DJL equation and the eKdV equation are reduced by $9.88 \%$ and $17.96 \%$, respectively, as shown in Fig. 3. Overall, the reduction in energy leads to the attenuation of the amplitude of the ISW, which in turn reduces the wave speed. Except for the difference in initial fields, the grid sizes, time step, turbulence model, and other features are the same. Therefore, the initial stage of ISWs generated by the eKdV equation leads to excessive energy loss compared with those generated by the DJL equation. From the above analysis of the velocity field, we know that the method of initializing the field with the eKdV equation requires a period of movement before the jump of the velocity field develops into a field with continuous changes in velocity. In addition, the DJL equation, as a fully nonlinear model, can better reflect its superiority for internal waves with strong nonlinearity. Therefore, the wave generation of the subsequent numerical cases in this paper adopts the method of initializing the field with the DJL equation.

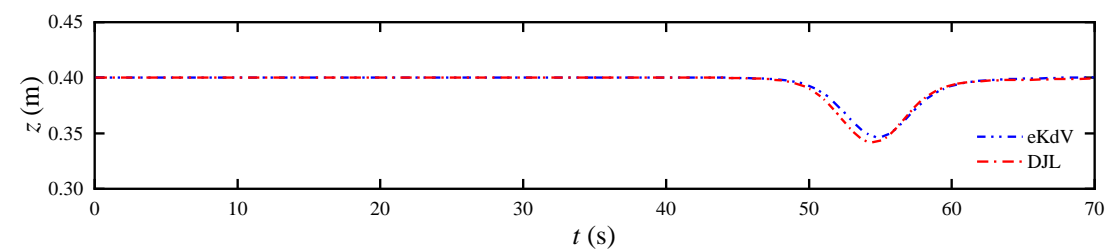

Figure 3: Time series of the interface displacement. The probe was $10 \mathrm{~m}$ away from the initial ISW. 


\section{Grid independence of the ISW simulation}

These grid independence tests were performed in the horizontal and vertical directions by applying meshes of different sizes. The sizes of the mesh determined in this paper are calculated based on the amplitude of the ISW and a characteristic length determined through the integration of the wave profile (Michallet and Ivey, 1999)

$$
L=\frac{1}{a} \int_{-\infty}^{\infty} \zeta(x) d x
$$

where $\zeta$ is the isopycnal vertical displacement and $a$ is the ISW amplitude.

To determine the appropriate mesh size, the propagation of ISWs on flat bottoms is calculated, and the numerical results are compared with the DJL theoretical solution. We set up a numerical simulation, which includes a tank that is $50 \mathrm{~m}$ long, $0.5 \mathrm{~m}$ wide and has a water depth of $0.5 \mathrm{~m}$. The depths of the upper $\left(h_{1}\right)$ and lower $\left(h_{2}\right)$ layers are $0.1 \mathrm{~m}$ and $0.4 \mathrm{~m}$, respectively, the densities of the upper and lower layers are $1000 \mathrm{~kg} / \mathrm{m}^{3}$ and $1030 \mathrm{~kg} / \mathrm{m}^{3}$, respectively, the location of the centre of the pycnocline $\left(z_{p y c}\right)$ is $0.4 \mathrm{~m}$, and the pycnocline thickness $\left(d_{p y c}\right)$ is $0.05 \mathrm{~m}$ vertically, the ISW amplitude $(a)$ is $0.065 \mathrm{~m}$. The measuring point $\mathrm{P}$ is set at a position $10 \mathrm{~L}$ away from the initial ISW. The sponge layer on both sides, whose length is the double wave characteristic length, has been checked to properly dissipate the reflected wave. Slip boundary conditions are applied to the bottom and both sides, while cyclic boundary conditions are assigned to the inlet and outlet boundaries. The top boundary is a rigid lid. The boundary conditions related to the density field are no-flux boundary conditions.

\subsection{Grid independence in the horizontal direction}

First, we analyse the grid independence in the horizontal direction, with a constant cell height of $\Delta z$ $=a / 20 \mathrm{~m}$. Fig. 4 shows the results of the comparison of the waveform and decay rate in the horizontal direction at probe P1 with the ISWFoam using a wide range of grid configurations. The results show a negligible difference in the waveform when the mesh size is less than $L / 40$, so it is difficult to accurately analyse the grid independence just by the waveform. A traditional decay rate parameter is adopted, namely $\delta=\left(a_{\text {probe }}-a_{\text {initial }}\right) / a_{\text {initial }}$, where $a_{\text {initial }}$ is the ISW amplitude value at the initial moment, $a_{\text {probe }}$ is the ISW amplitude value of the probe $10 L$ away from the initial ISW. Fig. 4(b) shows the relationship between the decay rate of the ISW amplitude and the grid quantity per unit length for different mesh sizes. As shown in Fig. 4(b), the decay rate of the ISW amplitude tends to be smooth as the grid number 
351 per unit length increases to $160(\Delta x=L / 150)$, and then the increase in the grid quantity has a relatively small effect on the decay rate. Therefore, for ISWFoam developed in this paper, we suggest that the dimensions of the horizontal grid are $L / 150$.
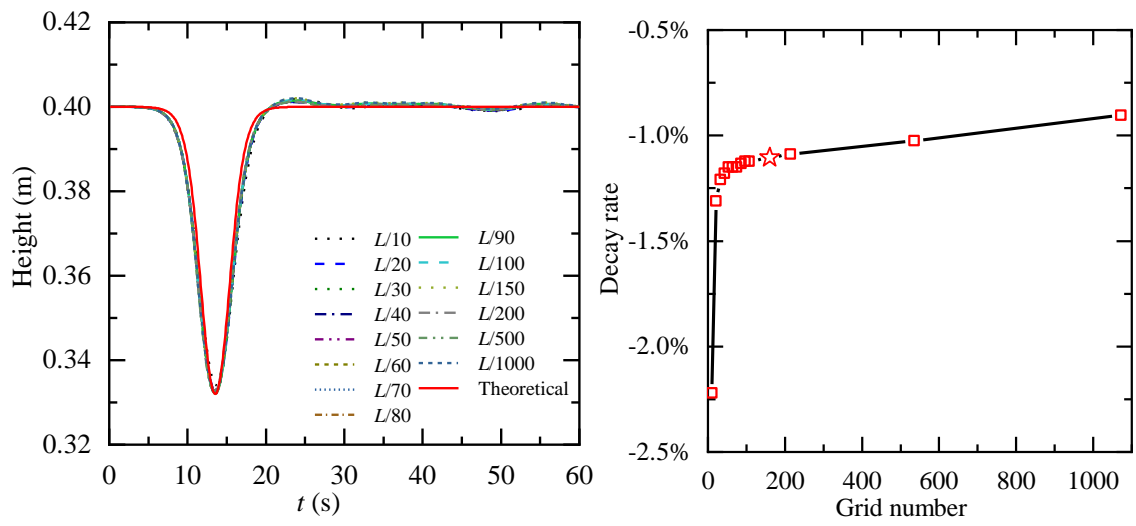

Figure 4: Grid independence in the horizontal direction at probe P1: (a) comparison of waveform and (b) decay rate.

\subsection{Grid independence in the vertical direction}

Second, we analyse the grid independence in the vertical direction, with a constant cell width of $\Delta x$ $=L / 150 \mathrm{~m}$. Fig. 5 shows the results of a comparison of the waveform and decay rate of the ISW amplitude in the vertical direction at probe P1 with the ISWFoam using a wide range of grid configurations. The results also show a negligible difference in the waveform when the mesh size is less than $a / 10$, so it is difficult to accurately analyse the grid independence just by the waveform. As shown in Fig. 5(b), the decay rate of the ISW amplitude decreases as the grid quantity increases in a wave height range before the numerical oscillation occurs. Here, we assume that the grid size with the decay rate of the ISW amplitude less than one percent is the appropriate vertical grid size; namely, the vertical grid size is $a / 25$ $\mathrm{m}$. Therefore, for ISWFoam developed in this paper, we suggest that the dimensions of the vertical grid be $a / 25$. 

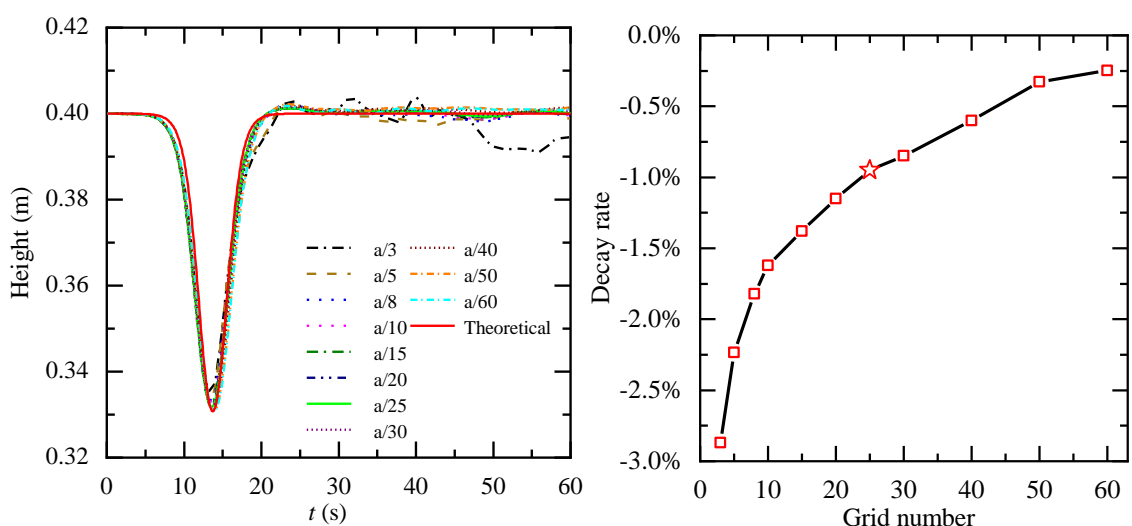

Figure 5: Grid independence analysis in the vertical direction at probe P1: (a) comparison of waveform and (b) decay rate.

Finally, for ISWFoam developed in this paper, we suggest that the dimensions of the horizontal grid are $L / 150$, while the vertical grid is $a / 25$.

\section{Model verification and results}

To verify the numerical model of the ISWs, the propagation of ISWs on a flat bottom section, submerged triangular ridge and slopes is calculated, and the numerical results are compared with the corresponding experimental results. To verify the correctness of Coriolis code implantation and reflect the role of local mesh refinement, the propagation of ISWs on a flat bottom section of actual ocean scale and a Gaussian ridge is calculated.

\subsection{ISW propagating on a flat bottom section}

\subsubsection{Experimental data used}

In this section, ISWFoam is verified by employing ISWs propagating on a flat bottom section with Case Flat_4 in the continuously stratified laboratory experiment described in Hsieh et al. (2014). The physical dimensions and ultrasonic probe locations in the experiments of Hsieh et al. (2014), as shown in Fig. 6, are adopted to establish the numerical computation domain. We set up a numerical tank of the experiment of Hsieh and co-authors, which includes a tank that is $15 \mathrm{~m}$ long, $0.5 \mathrm{~m}$ wide and has a stable water depth of $0.5 \mathrm{~m}$; the fluid densities of the upper $\left(\rho_{1}\right)$ and lower $\left(\rho_{2}\right)$ layers are $996 \mathrm{~kg} / \mathrm{m}^{3}$ and 1030 $\mathrm{kg} / \mathrm{m}^{3}$, respectively; the ISW amplitude $(a)$ is $0.068 \mathrm{~m}$; the location of the centre of the pycnocline $\left(z_{p y c}\right)$ is $0.4 \mathrm{~m}$, the pycnocline thickness $\left(d_{p y c}\right)$ is $0.04 \mathrm{~m}$ vertically, and the depths of the upper $\left(h_{1}\right)$ and lower 
$\left(h_{2}\right)$ layers are $0.1 \mathrm{~m}$ and $0.4 \mathrm{~m}$, respectively. The grid is uniform in the $x$-direction, $y$-direction and $z$ direction, and the sizes are $\Delta x=1.5 \times 10^{-2} \mathrm{~m}, \Delta y=1.5 \times 10^{-2} \mathrm{~m}$ and $\Delta z=2.72 \times 10^{-3} \mathrm{~m}$, respectively. The sponge layer on both sides, whose length is double wave characteristic length, has been checked to properly dissipate the reflected wave. Slip boundary conditions are applied to the bottom and both sides, rigid lid. The boundary conditions related to the density field are no-flux boundary conditions.

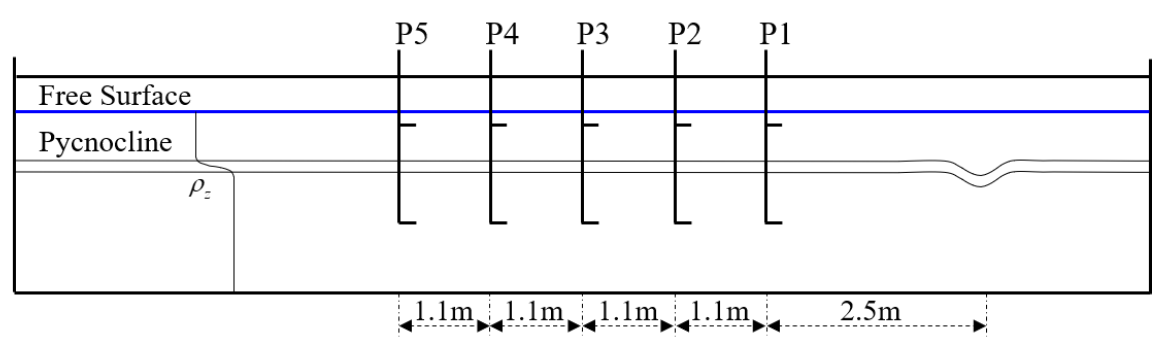

Figure 6: Schematic diagram of probe position (P1-P5) (Hsieh et al. (2014)).

\subsubsection{Comparisons between the numerical and experimental results}

Fig. 7 shows the density contours at three different times from Case Flat_4 in the laboratory experiment of Hsieh and coworkers, showing the stable evolution of an ISW. The results also show the realistic evolution of the thickening of the pycnocline after ISW propagation because of convection and diffusion. At the same time, the propagation of the ISW is stable and unbroken.
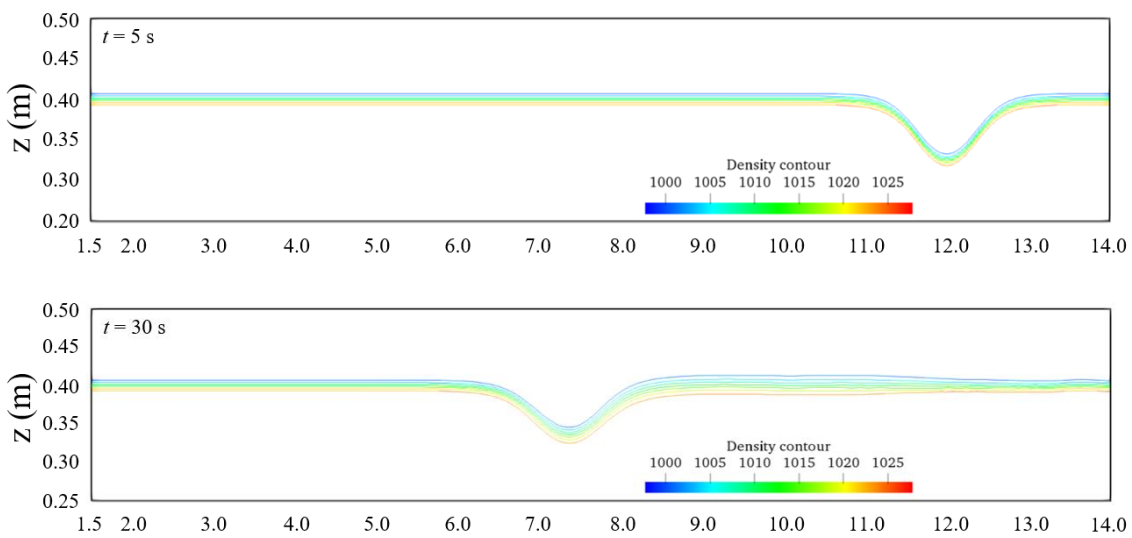
404

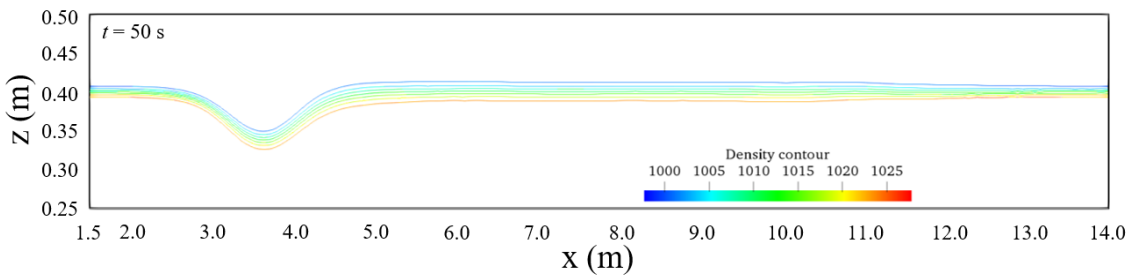

Figure 7: Density contours at different moments.

To further verify the model, the waveform is compared between the numerical simulations and the experimental measurements, and the measurement point selection is the same as the experimental setting, as shown in Fig. 6. Fig. 8 shows the comparison results between the waveform simulated by ISWFoam and the experimental results at probes P1-P5. Fig. 8 shows that the results of the numerical simulations agree with the experimental results (red circle). Notably, the laboratory wave height at the probe P1 measurement point is greater than the numerical simulation results, and the wave surface of the laboratory wave is not smooth, which is caused by the wave generation method using the gravity collapse mechanism in the laboratory. In general, the model developed in this paper can simulate the generation and evolution of ISWs in continuously stratified fluids.
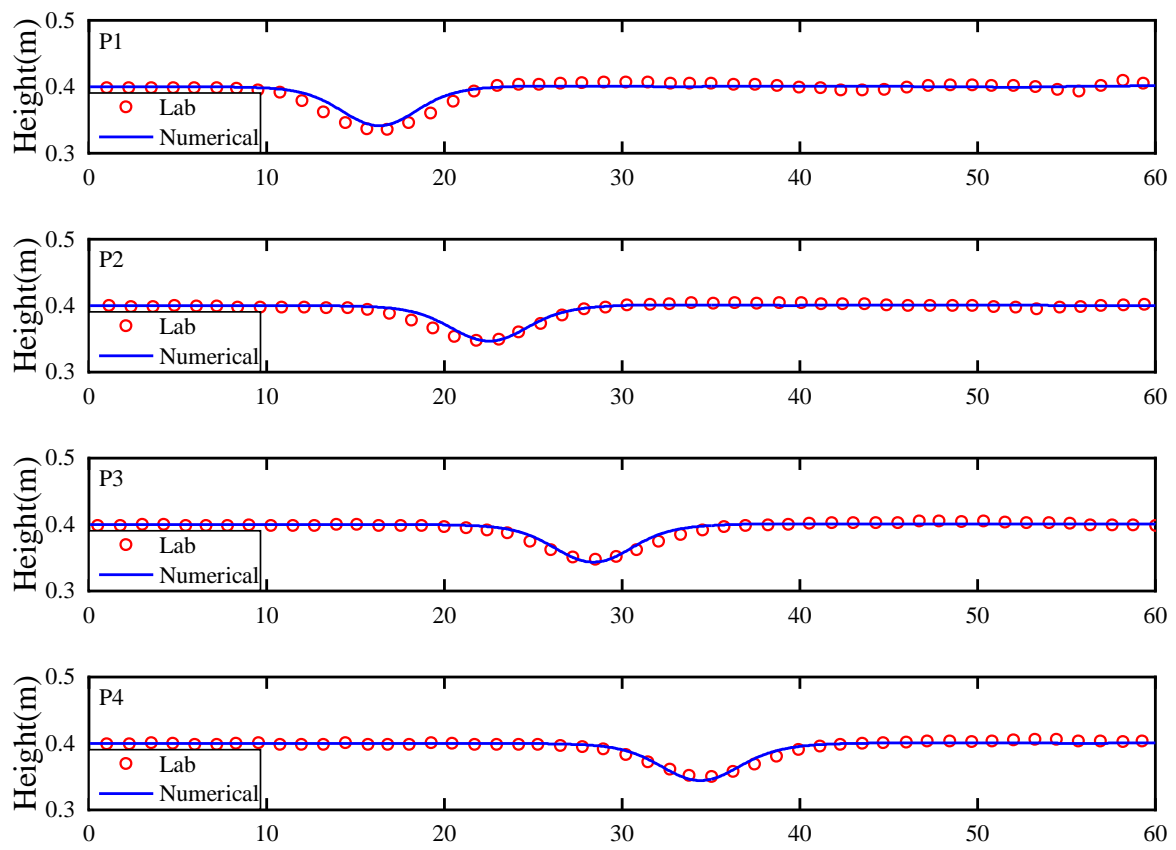


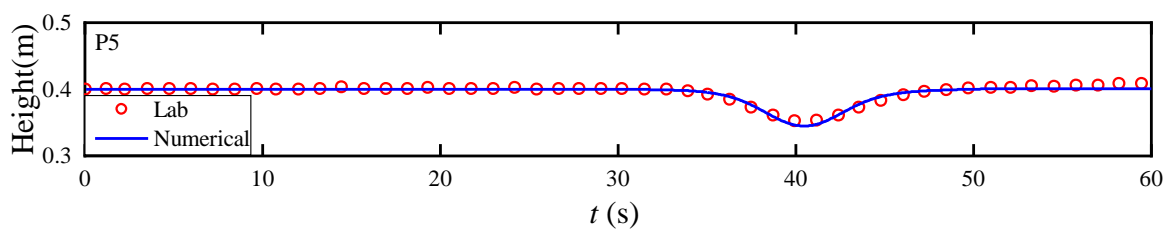

Figure 8: Comparison of the waveform between the experimental results and numerical simulation results at probes P1-P5.

\subsection{ISW propagating over a submerged triangular ridge}

\subsubsection{Experimental data used}

In this section, the validation of the numerical model is conducted through an ISW propagating over a submerged triangular ridge with the continuously stratified experiments described in Hsieh et al. (2015). The laboratory tank is $12 \mathrm{~m}$ long and has a stable water depth of $0.5 \mathrm{~m}$, with which the fluid system has a finite thickness of the pycnocline. The specific experimental parameters used for validation of ISWFoam include the various depths of the upper $\left(h_{1}\right)$ and lower $\left(h_{2}\right)$ layers; the fluid density of the upper $\left(\rho_{1}\right)$ and lower $\left(\rho_{2}\right)$ layers of $996 \mathrm{~kg} / \mathrm{m}^{3}$ and $1030 \mathrm{~kg} / \mathrm{m}^{3}$, respectively; the ISW amplitude $(\alpha=0.056 \mathrm{~m})$; the location of the centre of the pycnocline $\left(z_{p y c}=0.4 \mathrm{~m}\right)$; the thickness of the pycnocline $\left(d_{p y c}=0.04 \mathrm{~m}\right.$ vertically); the height of the isosceles triangular ridge ( $h_{\mathrm{s}}=0.30 \mathrm{~m}$ vertically); and the slope angle of the ridge for $\alpha=45^{\circ}$. The physical dimensions, and ultrasonic probe locations in the experiments of Hsieh et al. (2015), as shown in Fig. 9, are adopted to establish the numerical computation domain.

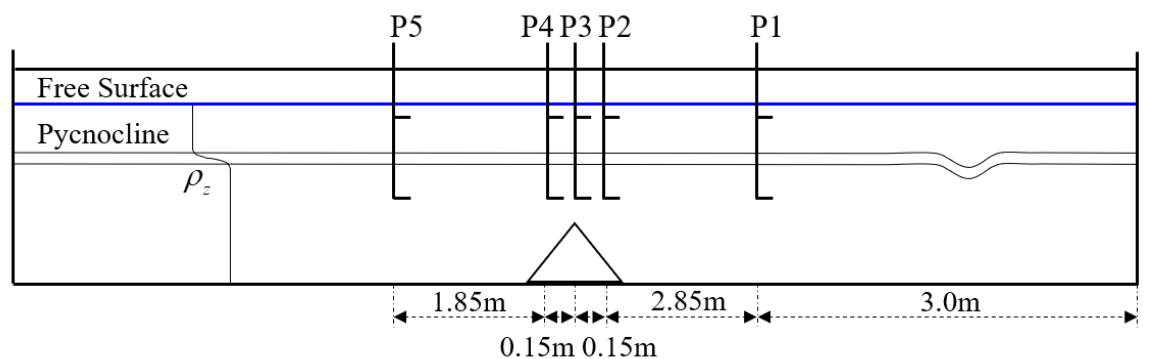

Figure 9: Schematic illustration of the laboratory setup and the locations of the probes (Hsieh et al. (2015)).

\subsubsection{Numerical implementation}

The numerical tank is designed to reproduce the experiment described in Fig. 9. The unstructured grid and local mesh refinement based on the finite volume method are used to construct the computational domain and discretize the governing equations. The grid is uniform in the $x$-direction, $y$-direction and $z$ - 
direction, and the sizes are $\Delta x=2 \times 10^{-3} \mathrm{~m}, \Delta y=2 \times 10^{-3} \mathrm{~m}$ and $\Delta z=2 \times 10^{-3} \mathrm{~m}$, respectively. The precise slope, as shown in Fig. 10. The sponge layer on both sides, whose length is double the wave characteristic length defined through integration of the wave profile in Section 3 for this case, has been checked to absorb the reflected wave well. A rigid wall conditions is applied to both sides, while the slip and slip conditions are assigned to the bottom and the surface of the submerged ridge boundaries, respectively. boundary conditions related to the density field are no-flux boundary conditions.

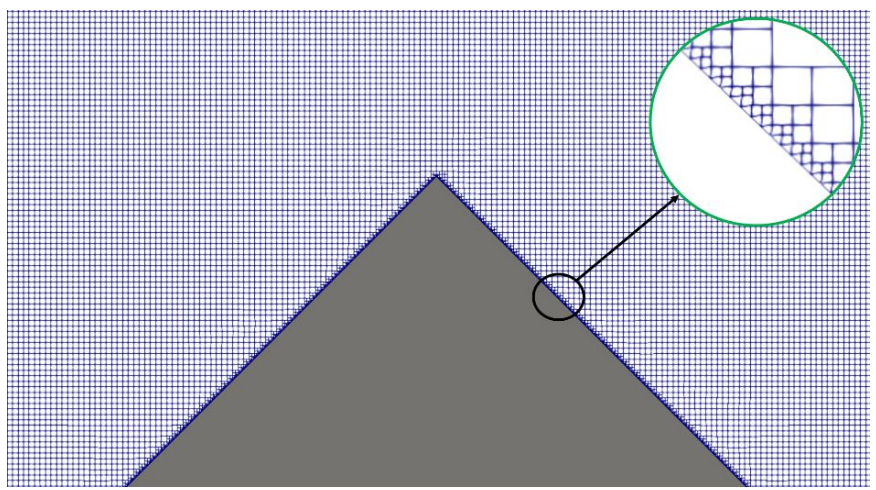

Fig. 10. Schematic of the mesh

\subsubsection{Comparisons between the numerical and experimental results}

Fig. 11 shows the comparison results between the waveform calculated by ISWFoam and the experimental results at probes P1-P5. In each subplot, the results of the numerical simulations (blue line) are found to be in good agreement with the experimental results (red circle). From Fig. 11 (a), the numerical simulation result of the probe P1 measurement after $20 \mathrm{~s}$ is different from the experimental results, which is caused by the different ISW generation methods. For the experimental results, the first large leading ISW is formed via the gravity collapse mechanism, which is trailed by a train of smallamplitude mode-one waves that is generated due to shear instabilities. However, the initialization method used to generate an initial ISW for the numerical simulation in this paper is more stable than the gravity collapse mechanism, so the rear part of the ISW is relatively flat compared to the experimental results for probe P1. In Fig. 11, the waveform of the ISW gradually evolves towards a flat waveform due to the interaction between the ISW and the ridge. In general, the model developed in this paper can simulate the interaction between ISWs and structures. 

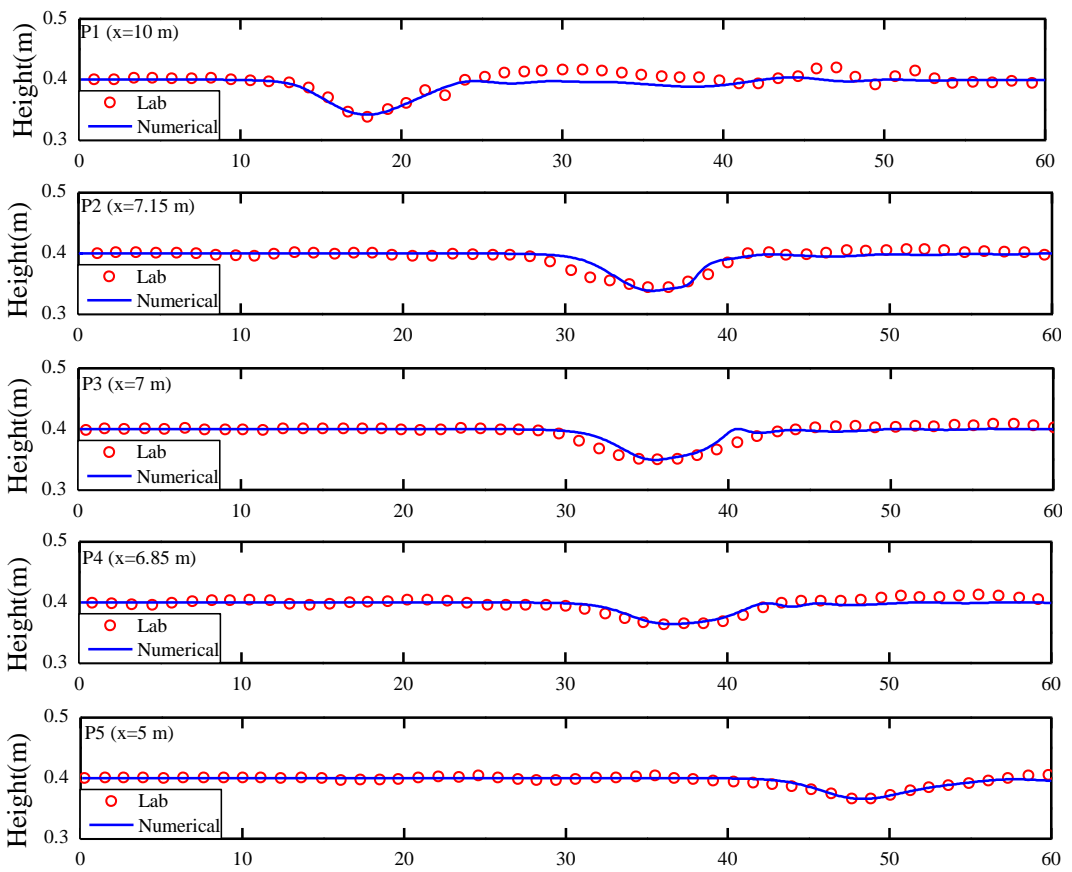

Figure 11: Comparison of the waveform between the experimental results in Hsieh et al. (2015) and numerical

\subsection{ISW propagating on a slope}

To verify the ability and accuracy of simulating the ISW breaking of the numerical model, two continuously stratified laboratory experiments (12 and 15) of Michallet and Ivey. (1999) are chosen for the simulation in this section. The experimental setup is represented schematically in Fig. 12. We set up a numerical tank of the experiment of Michallet and Ivey. (1999), which includes a tank is $L=4.2 \mathrm{~m}$ long, $0.25 \mathrm{~m}$ wide and has a water depth of $0.15 \mathrm{~m}$. The layer thickness ratio $(h / H)$ varies from $0.60 \sim 0.91$. A linear slope $s=0.214$ starts at $0.7 \mathrm{~m}$ from the right end of the tank for experiments 12 and 15 . The grid is uniform in the $x$-direction, $y$-direction and $z$-direction, and the sizes are $\Delta x=2.5 \times 10^{-3} \mathrm{~m}, \Delta y=2.5 \times 10^{-}$

$478{ }^{3} \mathrm{~m}$ and $\Delta z=1.25 \times 10^{-3} \mathrm{~m}$, respectively. The precise grid describing the slope section is $\Delta x=\Delta y=6.25 \times 10^{-}$

$479{ }^{4} \mathrm{~m}$ and $\Delta z=3.125 \times 10^{-4} \mathrm{~m}$ at the slope. 


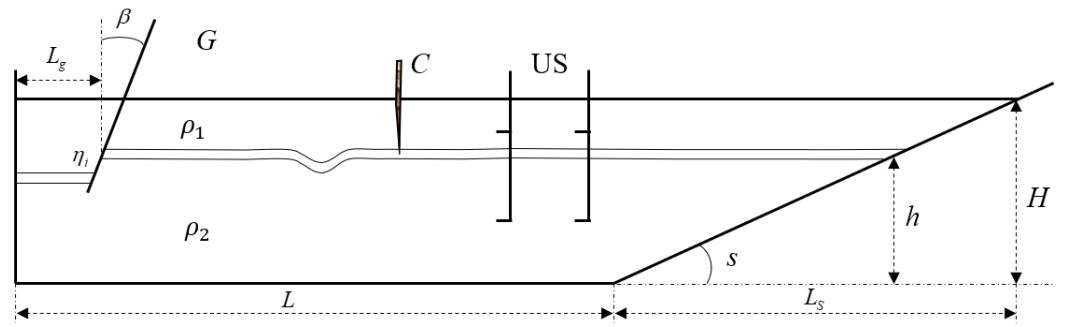

Figyre 12: Schematic diagram of the laboratory setup. "C" and "US" represent the experimental device at probes.

The sponge layer on the left side, whose length is the double wave characteristic length, is checked to properly dissipate the reflected wave. Slip boundary conditions are applied to the bottom and both sides, while slip boundary conditions are assigned to the top boundaries. The boundary conditions related to the density field are no-flux boundary conditions.

The vertical profile of the initial density is given by a hyperbolic tangent function profile

$$
\bar{\rho}(z)=\rho_{1}+\frac{\Delta \rho}{2}\left\{1+\tanh \left[\frac{-\left(z-z_{p y c}\right)}{d_{p y c}}\right]\right\}
$$

where $z$ is the vertical position, $\rho_{1}=1 \times 10^{3} \mathrm{~kg} / \mathrm{m}^{3}$ is the base density field, $\Delta \rho$ is the change in the density, $z_{p y c}$ is the location of the centre of the pycnocline, and $d_{p y c}$ is the thickness of the pycnocline.

\subsubsection{Case one and results}

The first case of model verification is experiment 12 of Michallet and Ivey. (1999) in this section. The layer thickness ratio $(h / H)$ is 0.84 , and the density change $(\Delta \rho)$ value is $14 \mathrm{~kg} / \mathrm{m}^{3}$. Fig. 13 presents

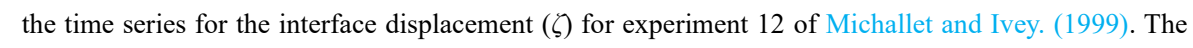
results indicate reasonably good agreement between the time series of the simulated interface displacement and that of the laboratory results. The first trough centred around $t=25 \mathrm{~s}$ represents the incident ISW propagating the probe $99.8 \mathrm{~cm}$ away from the start of the slope. The second trough centred at approximately $t=87 \mathrm{~s}$ represents the reflected ISW at the generation side, which has a smaller amplitude and a longer wavelength than the incident ISW as the energy in the wave decreases. As shown in Fig. 13, the smooth waveform of the incident ISW of the numerical simulation indicates that the initialization method of wave generation in this paper is more stable than the experiment. 
https://doi.org/10.5194/gmd-2021-102

Preprint. Discussion started: 27 May 2021

(c) Author(s) 2021. CC BY 4.0 License.

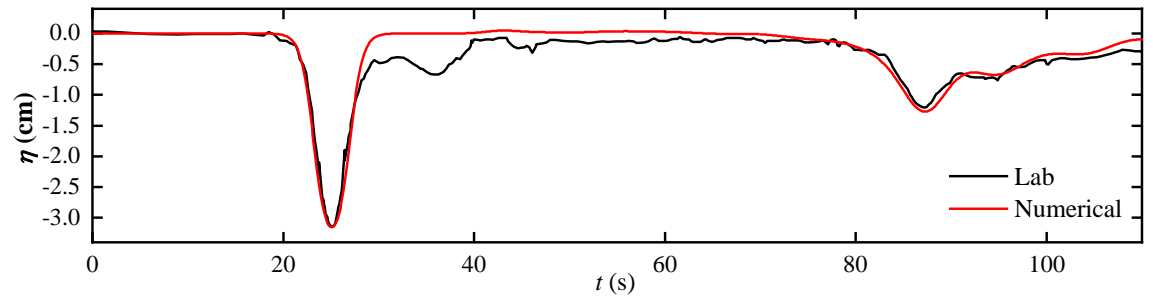

Figure 13: Time series of the interface displacement. The probe was $99.8 \mathrm{~cm}$ away from the start of the slope.

Fig. 14 shows a comparison of ISWFoam results and the experimental observations of the velocity field associated with the ISW run-up process along the slope. The model effectively reproduces laboratory tests, such as the intensity and direction of the velocity field, the location of the vortices, and the occurrence of boundary-layer separation beneath the ISW. Therefore, the model developed in this paper can reflect the ISW breaking phenomenon during the propagation of ISWs along the slope.
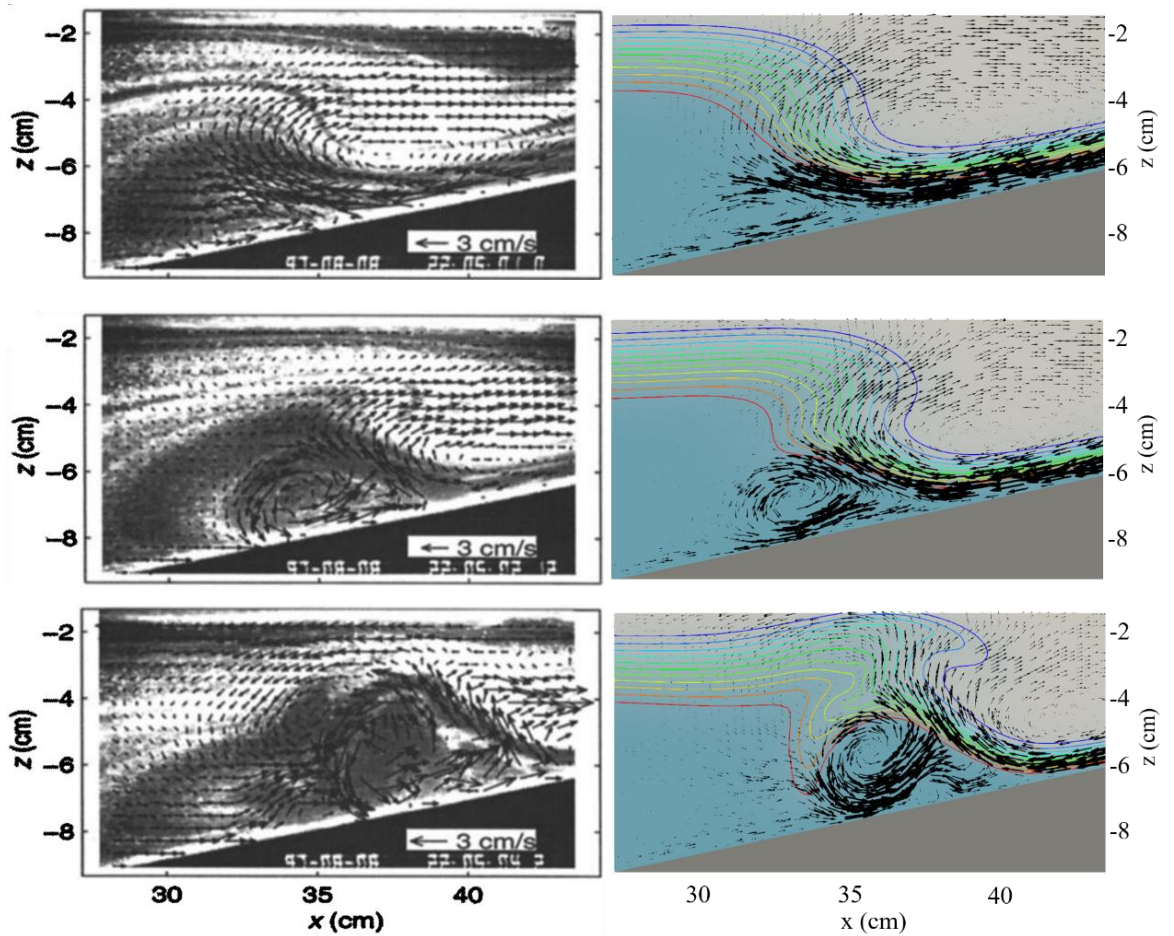

Figure 14: Comparison of the velocity field between the experimental observation results in Michallet and Ivey. (1999) (left) and numerical simulation results (right).

\subsubsection{Case two and results}

Another laboratory experiment that more clearly shows the ISW breaking phenomenon from 
Experiment 15 of Michallet and Ivey. (1999) is used to verify the numerical model presented in this paper, and the corresponding numerical case is set corresponding to it. The layer thickness ratio $(h / H)$ is 0.77 , and the density change $(\Delta \rho)$ value is $47 \mathrm{~kg} / \mathrm{m}^{3}$. The wave amplitude and phase velocity at the slope calculated by ISWFoam are $2.71 \times 10^{-3} \mathrm{~m}$ and $10.83 \times 10^{-1} \mathrm{~m} / \mathrm{s}$, which fit well with the experimental results of $2.7 \times 10^{-3} \mathrm{~m}$ and $10.8 \times 10^{-1} \mathrm{~m} / \mathrm{s}$.

Fig. 15 shows the results of the numerical simulations of the ISWs propagating along the slope and wave breaking using ISWFoam. As the ISW propagates to the slope, according to the conservation of mass, the upper fluid forward and the downward velocity of the lower fluid increasing along the slope results in the formation of a thin boundary layer, as shown in Fig. 15(a), (b), and (c). At the same time, the amplitude of the ISW increases, and the rear of the ISW gradually becomes very steep but does not overturn. With the development of the ISW, the rear waveform of the ISW cannot maintain its stability and overturns forward, resulting in wave breaking, as shown in Fig. 15(d). After wave breaking occurs, the denser lower layer flow accelerates into the less dense upper layer flow, forming a mixture region, as shown in Fig. 15(e). After the lower layer flow is drawn downward from beneath the ISW, a mixing region comprised of vortices is pushed upwards along the slope while the leading waveform is reflected,

for wave breaking.
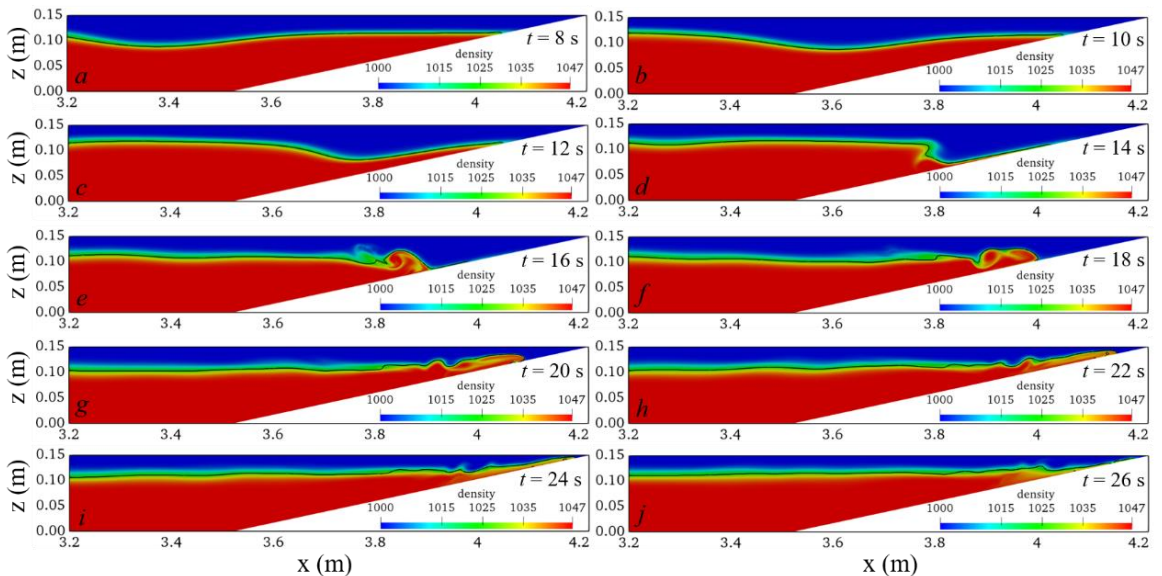

Figure 15: Temporal and spatial variations in the ISWs breaking calculated using ISWFoam (the black line represents the waveform).

For comparison with the flow visualization image of the experiment, a specified thickness of the 

shown in Fig. 16.

Fig. 16 compares the ISWFoam results and the experimental results of Michallet and Ivey. (1999) before, during, and after ISW breaking. The results indicate that some main features of the laboratory tests are reasonably well reproduced by ISWFoam, such as the profile of ISW, the location of the wave breaking point, ISW arrival time, and spatial and temporal changes in the mixture region. Therefore, the model developed in this paper can accurately simulate the ISW breaking phenomenon during the propagation of ISWs along the slope.
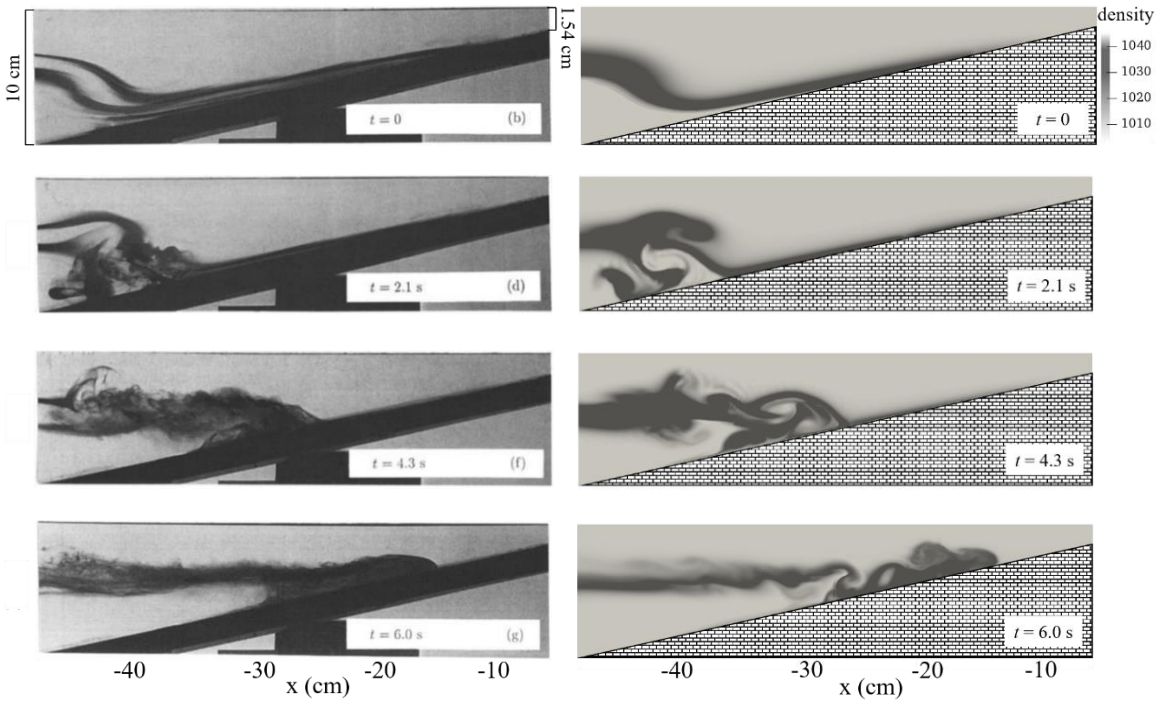

Figure 16: Comparison of the density fields between the experimental observation results in Michallet and Ivey,

(1999) (left) and the numerical simulation results (right).

\section{1}

\subsection{Coriolis force analysis}

Notably, the previous verifications are all laboratory-scale verifications, thus, it is difficult to clearly see the influence of the Coriolis force on ISWs. To verify the correctness of the Coriolis force added in the code, we set up a 3D numerical tank, which includes a tank that is $15 \mathrm{~km}$ long, $1 \mathrm{~km}$ wide ( $y$ direction from $-500 \mathrm{~m}$ to $500 \mathrm{~m})$ and has a water depth of $300 \mathrm{~m}$. The depths of the upper $\left(h_{1}\right)$ and lower $\left(h_{2}\right)$ layers are $50 \mathrm{~m}$ and $250 \mathrm{~m}$, respectively, the densities of the upper and lower layers are $1022 \mathrm{~kg} / \mathrm{m}^{3}$ and 1028 $\mathrm{kg} / \mathrm{m}^{3}$, respectively, the location of the centre of the pycnocline $\left(z_{p y c}\right)$ is $250 \mathrm{~m}$ vertically, and the pycnocline thickness $\left(d_{p y c}\right)$ is $5 \mathrm{~m}$ vertically, the ISW amplitude $(a)$ is $40 \mathrm{~m}$. The grid gradually changes 
559 from $\Delta x=100 \mathrm{~m}$ to $\Delta x=10 \mathrm{~m}$ in the $x$-direction, the grids in the $y$-direction are uniform with a constant 560 cell width of $\Delta y=10 \mathrm{~m}$, and the grids in the $z$-direction are non-uniform, with a minimum cell height of $561 \Delta z=2.5 \mathrm{~m}$ near the interface of the ISW. To see the influence of the Coriolis force more clearly, the 562 Coriolis force parameter $(\Omega)$ is expanded 5 times, to $3.65 \times 10^{-4} \mathrm{~s}^{-1}$. The sponge layer on both sides, whose 563 length is the double wave characteristic length, has been checked to properly dissipate the reflected wave. 564 Slip boundary conditions are applied to the bottom and both sides, while cyclic boundary conditions are 565 assigned to the inlet and outlet boundaries. The top boundary is a rigid lid. The boundary conditions 566 related to the density field are no-flux boundary conditions.

Figs 17 and 18 show the two-dimensional (2D) and three-dimensional (3D) waveform diagrams when the ISW propagates for 5000s. The figures show that under the influence of the Coriolis force, asymmetry is caused in the front and back wave surfaces, and the height difference is approximately 6 $\mathrm{m}$. Since this summary only verifies the correctness of the Coriolis force implanted in the code, there is no further quantitative analysis of the influence of the Coriolis force on ISWs. In general, ISWFoam can effectively reflect the influence of the Coriolis force. At the same time, the influence of the Coriolis force cannot be ignored when performing large-scale simulations.

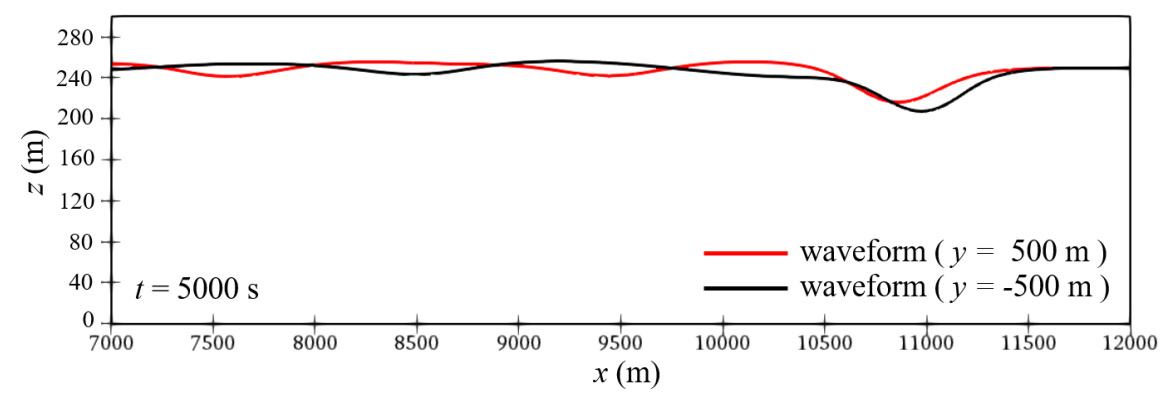




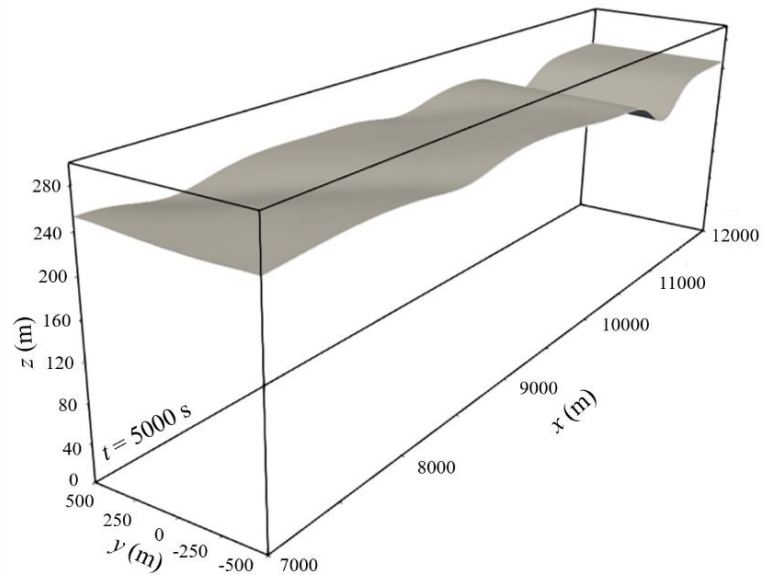

Figure 18: Schematic of the three-dimensional waveform.

\subsection{ISW propagating over a 3D Gaussian ridge}

The ISWFoam model with unstructured grids and local mesh refinement can accurately simulate the interaction between ISWs and complex structures and topography. However, the terrain in the previous cases is relatively simple, and it is difficult to see such characteristics. Therefore, we designed a case of an ISW propagating over a 3D Gaussian ridge. The 3D Gaussian ridge is obtained by rotating a 2D Gaussian ridge

$$
z=a e^{-(x / l)^{2}}
$$

where $a$ is the ridge amplitude, and $l$ is the standard deviation.

With $a=5 \mathrm{~m}$ and $l=0.5$, we can obtain a $2 \mathrm{D}$ Gaussian ridge with a height of $5 \mathrm{~m}$ and a bottom width of $2 \mathrm{~m}$. Subsequently, the 3D Gaussian ridge can be obtained after a vertical rotation of 180 degrees.

We set up a 3D numerical tank, which includes a tank that is $150 \mathrm{~m}$ long, $20 \mathrm{~m}$ wide ( $y$-direction from $-10 \mathrm{~m}$ to $10 \mathrm{~m})$ and has a water depth of $6 \mathrm{~m}$. The depths of the upper $\left(h_{1}\right)$ and lower $\left(h_{2}\right)$ layers are $1 \mathrm{~m}$ and $5 \mathrm{~m}$, respectively, the densities of the upper and lower layers are $1000 \mathrm{~kg} / \mathrm{m}^{3}$ and $1030 \mathrm{~kg} / \mathrm{m}^{3}$, respectively, the location of the centre of the pycnocline $\left(z_{p y c}\right)$ is $5 \mathrm{~m}$, and the pycnocline thickness $\left(d_{p y c}\right)$ is $0.1 \mathrm{~m}$ vertically, the ISW amplitude $(a)$ is $1 \mathrm{~m}$. The Gaussian ridge is located at $80 \mathrm{~m}$ horizontally. The grid is gradually changed from $\Delta x=5 \mathrm{~m}$ to $\Delta x=0.2 \mathrm{~m}$ in the $x$-direction, the grids in the $y$-direction are uniform with a constant cell width of $\Delta y=0.2 \mathrm{~m}$, and the grids in the $z$-direction are non-uniform, with a minimum cell height of $\Delta z=0.05 \mathrm{~m}$ near the interface of the ISW. The precise grid described the 3D 
Gaussian ridge section as $\Delta x=1.25 \times 10^{-2} \mathrm{~m}, \Delta y=1.25 \times 10^{-2} \mathrm{~m}$ and $\Delta z=6.25 \times 10^{-3} \mathrm{~m}$, as shown in Fig 19 .

The sponge layer on both sides, whose length is the double wave characteristic length, has been checked

to properly dissipate the reflected wave. Slip boundary conditions are applied to the bottom and both is a rigid lid. The boundary conditions related to the density field are no-flux boundary conditions.

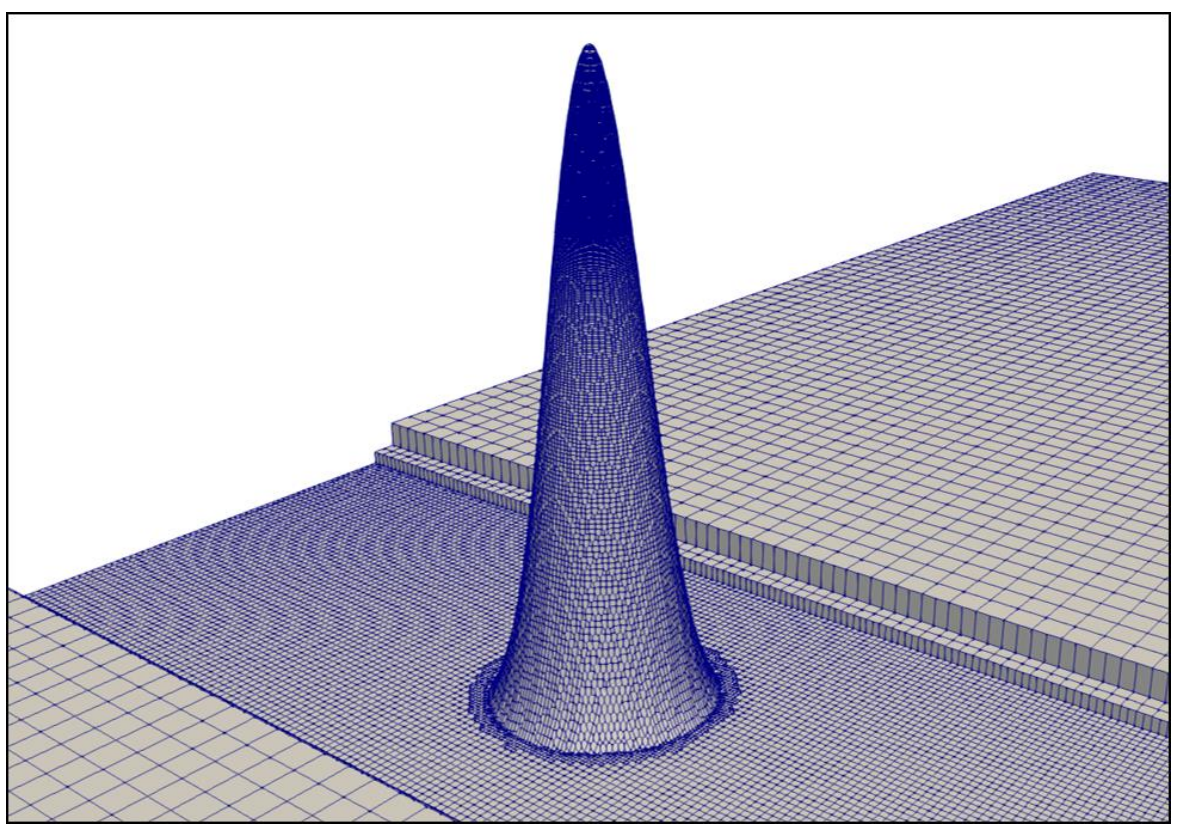

Figure 19: Schematic of the local refinement of the grid.

Fig. 20. shows the temporal and spatial variations in the ISWs propagating over a 3D Gaussian ridge. The ISW reaches the Gaussian ridge, causing the wave surface in front of the ridge to decrease, and the wave surface behind the ridge to climb up the ridge, as shown in Fig. 20(a). Due to being obstructed by the Gaussian ridge, flow around a ridge and wave surface uplift are generated on both sides of the Gaussian ridge (perpendicular to the direction of wave propagation), as shown in Fig. 20(b). As the ISW propagated over the Gaussian ridge, the wave surface climbed along the ridge, and at the same time, low velocity was generated behind the ridge, as shown in Fig. 20(c). Since the top of the ridge is in the pycnocline, there will be a low velocity area behind the ridge for a period of time after the ISW passes, as shown in Fig. 20(d). In general, the ISWFoam model with unstructured grids and local mesh refinement can simulate the interaction between ISWs and complex structures and topography. 

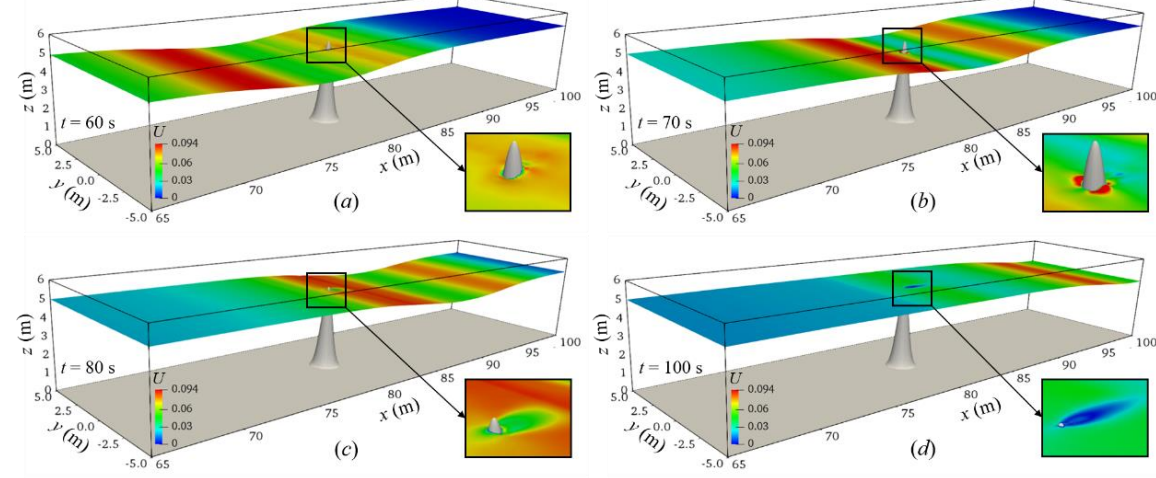

Figure 20: Temporal and spatial variation in the ISWs propagating over a 3D Gaussian ridge.

\section{Conclusions}

In this paper, a numerical model referred to as ISWFoam with a modified $k$ - $\omega$ SST model, established by combining the density transport equation with a fully three-dimensional (3D) NavierStokes equation, is developed to simulate ISWs in continuously stratified, incompressible, viscous fluids based on the finite volume method with unstructured grids and local mesh refinement of OpenFOAM. ISWFoam provides two initial wave generation methods to generate an ISW in continuously stratified fluids, including solving the weakly nonlinear models of the $\mathrm{eKdV}$ equation and the fully nonlinear models of the DJL equation. The verification process presents several applications, such as ISWs propagating on flat bottoms including laboratory scale and actual ocean scale, and ISWs over a submerged triangular ridge, a Gaussian ridge and slopes. The following conclusions were obtained as a result of this study.

ISWFoam using the finite volume method with unstructured grids and local mesh refinement can accurately simulate the generation and evolution of ISWs, the ISW breaking phenomenon and the interaction between ISWs and complex structures and topography. The method of initializing the ISW using weakly nonlinear eKdV equation models requires a period of movement before the jump of the velocity field develops into a field with continuous changes in velocity. The DJL equation wave generation method that considers the vertical velocity and the horizontal velocity along the vertical gradient is better than the $\mathrm{KdV}$ equation wave generation method that only provides the horizontal average velocity. Using ISWFoam to simulate an ISW with infinite wave length, the metric for the appropriate mesh size is given as follows: the dimensions of the horizontal grid are one-one hundred and 
The ISWFoam code developed in this article can be downloaded for free from

\section{Author contributions}

QZ and JL jointly developed this numerical method to calculate internal solitary waves in continuously stratified fluids. JL developed the code. TC performed the computations. QZ and JL jointly analysed the calculation results and wrote the paper together.

\section{Competing interest}

The authors of this paper declare that they have no conflicts of interest.

\section{Financial support}

\section{Reference}

Alford, M. H., Lien, R. C., Simmons, H., Klymak, J., Ramp, S., Yang, Y. J., Tang, D., and Chang, M. H.: Speed and evolution of nonlinear internal waves transiting the South China Sea, Journal of Physical Oceanography., 40, 1338-1355, doi:10.1175/2010JPO4388.1, 2010.

Alford, M. H., MacKinnon, J. A., Nash, J. D., Simmons, H., Pickering, A., Klymak, J. M., Beitzel, T.: Energy flux and dissipation in Luzon Strait: Two tales of two ridges, Journal of Physical Oceanography., 41, 2211-2222. doi:10.1175/JPOD11073.1, 2011.

Alford, M. H., Peacock, T., MacKinnon, J. A., Nash, J. D., Buijsman, M. C., Centurioni, L. R., Fu, K. H.: The formation and fate of internal waves in the South China Sea, Nature., 521, 65-69, doi:10.1038/nature14399, 2015.

Aghsaee, P., Boegman, L., Lamb, K. G.: Breaking of shoaling internal solitary waves, Journal of Fluid Mechanics., 659, 289. doi:10.1017/S002211201000248X, 2010.

Aghsaee, P., Boegman, L., Diamessis, P. J., Lamb, K. G.: Boundary-layer-separation-driven vortex shedding beneath internal solitary waves of depression, Journal of Fluid Mechanics., 690, 321, 
doi:10.1017/jfm.2011.432, 2012.

Apel, J., Ostrovsky, L., Stepanyants, Y., Lynch, J.F.: Internal Solitons in the Ocean, Technical Report

Woods Hole Oceanographic Institution., doi:10.1575/1912/1070, 2006.

Bardina, J.E., Coakley, T.J., and Huang, P.G.: Turbulence Modeling Validation, Testing, and Development, NASA TM 110446, NASA Ames Research Center, Moffett Field, Calif. doi: 10.2514/6.1997-2121, 1997.

Bell, J. B. and Marcus, D. L.: A second-order projection method for variable-density flows, Journal of Computational Physics., 101(2), 334-348, doi:10.1016/0021-9991(92)90011-M, 1992.

Brown, D. J. and Christie, D. R.: Fully nonlinear solitary waves in continuously stratified incompressible Boussinesq fluids, Physics of Fluids., 10(10), 2569-2586, doi:10.1063/1.869771, 1998.

Boegman, L. and Stastna, M.: Sediment resuspension and transport by internal solitary waves, Annual review of fluid mechanics., 51, 129-154, doi:10.1146/annurev-fluid-122316-045049, 2019.

Buijsman, M. C., McWilliams, J. C., and Jackson, C. R.: East-west asymmetry in nonlinear internal waves from Luzon Strait, Journal of Geophysical Research: Oceans., 115(C10), doi:10.1029/2009JC006004, 2010.

Cai, S., Xie, J., and He, J.: An overview of internal solitary waves in the South China Sea, Surveys in Geophysics., 33, 927-943, doi:10.1007/s10712-012-9176-0, 2012.

Cheng, M. H., Hwang, R. R., and Hsieh, C. M.: Numerical study on the transformation of an internal solitary wave propagating across a vertical cylinder, Applied Ocean Research., 95, 102016, doi:10.1016/j.apor.2019.102016, 2020.

Choi, W. and Camassa, R.: Fully nonlinear internal waves in a two-fluid system, Journal of Fluid Mechanics., 396, 1-36, doi:10.1017/S0022112099005820, 1999

Deshpande, S. S., Anumolu, L., and Trujillo, M. F.: Evaluating the performance of the two-phase flow solver interFoam, Computational Science. Discovery 5, 014016, 1-36, doi:10.1088/17494699/5/1/014016, 2012.

Diamessis, P. J., Domaradzki, J. A., and Hesthaven, J. S.: A spectral multidomain penalty method model for the simulation of high Reynolds number localized incompressible stratified turbulence, Journal of Computational Physics., 202(1), 298-322, doi:10.1016/j.jcp.2004.07.007, 2005.

Ding, W., Ai, C., Jin, S., Lin, J.: Numerical investigation of an internal solitary wave interaction with horizontal cylinders, Ocean Engineering., 208, 107430, doi:10.1016/j.oceaneng.2020.107430, 2020.

Dunphy, M., Subich, C., and Stastna, M.: Spectral methods for internal waves: indistinguishable density profiles and double-humped solitary waves, Nonlinear Processes in Geophysics., 18(3), 351-358, doi:10.5194/npg-18-351-2011, 2011.

Farmer, D. M.and Smith, J. D.: Tidal interaction of stratified flow with a sill in Knight Inlet, Deep Sea Research Part A. Oceanographic Research Papers., 27(3-4), 239-254, doi:10.1016/01980149(80)90015-1, 1980.

Fringer, O. B., Gerritsen, M., and Street, R. L.: An unstructured-grid, finite-volume, nonhydrostatic, parallel coastal ocean simulator, Ocean Modelling., 14(3-4), 139-173, 
Fu, D. M., You, Y. X., and Li, W., Numerical simulation of internal solitary waves with a submerged body in a two-layer fluid, Ocean Engineering (Haiyang Gongcheng)., 37(3), 38-44, 2009.

Gao, X. Y., You, Y. X., Wang, X., Li, W.: Numerical simulation for the internal solitary wave based on MCC theory, Ocean Engineering (Haiyang Gongcheng)., 30(4), 29-36, 2012.

Grimshaw, R., Pelinovsky, E., Talipova, T., Kurkina, O.: Internal solitary waves: propagation, deformation and disintegration, Nonlinear Processes in Geophysics., 17(6), 633-649, doi:10.5194/npg-17-633-2010, 2010.

Guo, C. and Chen, X.: A review of internal solitary wave dynamics in the northern South China Sea, Progress in Oceanography., 121, 7-23, doi:10.1016/j.pocean.2013.04.002, 2014.

Hallberg, R. and Rhines, P.: Buoyancy-driven circulation in an ocean basin with isopycnals intersecting the sloping boundary, Journal of Physical Oceanography., 26(6), 913-940, doi:10.1175/15200485(1996)026<0913:BDCIAO>2.0.CO;2, 1996.

Hallberg, R.: Stable split time stepping schemes for large-scale ocean modeling, Journal of Computational Physics., 135(1), 54-65, doi:10.1006/jcph.1997.5734, 1997.

Helfrich, K. R. and Melville, W. K.: Long nonlinear internal waves, Annu. Rev. Fluid Mech., 38, $395-$ 425, doi:10.1146/annurev.fluid.38.050304.092129, 2006.

Hsieh, C. M., Hwang, R. R., Hsu, J. R. C., Cheng, M. H.: Flow evolution of an internal solitary wave generated by gravity collapse, Applied Ocean Research., 48, 277-291, doi:10.1016/j.apor.2014.10.001, 2014.

Hsieh, C. M., Hwang, R. R., Hsu, J. R. C., Cheng, M. H.: Numerical modeling of flow evolution for an internal solitary wave propagating over a submerged ridge, Wave Motion., 55, 48-72, doi:10.1016/j.wavemoti.2014.12.008, 2015.

Hibiya, T.: The generation of internal waves by tidal flow over Stellwagen Bank, Journal of Geophysical Research: Oceans., 93, 533-542, doi:10.1029/JC093iC01p00533, 1988.

Hu, H. C., Tong, B. and Lou, S. Y.: Nonsingular positon and complexiton solutions for the coupled KdV system, Physics Letters A., 351, 403-412, doi:10.1016/j.physleta.2005.11.047, 2006.

Huthnance, J. M.: Waves and currents near the continental shelf edge, Progress in Oceanography., 10, 193-226, doi:10.1016/0079-6611(81)90004-5, 1981.

Issa, R. I.: Solution of the implicitly discretised fluid flow equations by operator-splitting, Journal of computational physics., 62(1), 40-65, doi:10.1016/0021-9991(86)90099-9, 1986.

Jasak, H.: Error Analysis and Estimation for the Finite Volume Method with Applications to Fluid Flows, Ph.D. thesis. Imperial College London, 1996.

Ko, D. S., Martin, P. J., Rowley, C. D., Preller, R. H.: A real-time coastal ocean prediction experiment for MREA04, Journal of Marine Systems., 69(1-2), 17-28, doi:10.1016/j.jmarsys.2007.02.022, 2008.

Kubota, T., Ko, D. R. S., and Dobbs, L. D.: Weakly-nonlinear, long internal gravity waves in stratified fluids of finite depth, Journal of Hydronautics., 12, 157-165, doi:10.2514/3.63127, 1978. 
Lamb, K. G.: Numerical experiments of internal wave generation by strong tidal flow across a finite amplitude bank edge, Journal of Geophysical Research: Oceans., 99, 843-864, doi:10.1029/93JC02514, 1994.

Lamb, K. G. and Yan, L.: The evolution of internal wave undular bores: comparisons of a fully nonlinear numerical model with weakly nonlinear theory, Journal of physical oceanography., 26(12), 27122734, doi:10.1175/1520-0485(1996)026<2712:TEOIWU>2.0.CO;2, 1996.

Lamb, K. G. and Nguyen, V. T.: Calculating energy flux in internal solitary waves with an application to reflectance, Journal of Physical Oceanography., 39, 559-580, doi:10.1175/2008JPO3882.1, 2009.

Lamb, K. G.: Energy and pseudoenergy flux in the internal wave field generated by tidal flow over topography, Continental Shelf Research., 27, 1208-1232, doi:10.1016/j.csr.2007.01.020, 2007.

Li, J. Y., Zhang, Q. H., and Chen. T. Q.: Numerical Simulation of Internal Solitary Wave in Continuously Stratified Fluid, Journal of Tianjin University (Science and Technology)., 54(02), 161-170, DOI:10.11784/tdxbz202001025, 2021.

Li, Q. and Farmer, D. M.: The generation and evolution of nonlinear internal waves in the deep basin of the South China Sea, Journal of Physical Oceanography., 41(7), 1345-1363, doi:10.1175/2011JPO4587.1, 2011.

Li, Z., You, Y. U., Zhe, S., Zang, J. M., LI, Z. H., Yu, Z. B.: CFD Simulation of Internal Solitary Wave Using the Volume-of-fluid Method within OpenFOAM, DEStech Transactions on Computer Science and Engineering, doi:10.12783/dtcse/mmsta2017/19617, 2017 (mmsta).

Long, R. R.: Some aspects of the flow of stratified fluids: I. A theoretical investigation,Tellus.,5(1), 4258, doi:10.3402/tellusa.v5i1.8563, 1953.

Lou, S. Y., Tong, B., Hu, H. C., Tang, X. Y.: Coupled KdV equations derived from two-layer fluids, Journal of Physics A: Mathematical and General., 39, 513, doi:10.1088/0305-4470/39/3/005, 2005.

Meng, Q. and Zhang, C.: A third-order KdV solution for internal solitary waves and its application in the numerical wave tank, Journal of Ocean Engineering and Science., 1(2), 93-108, doi:10.1016/j.joes.2016.03.002, 2016.

Menter, F. R., Kuntz, M., and Langtry, R.: Ten years of industrial experience with the SST turbulence model,Turbulence, heat and mass transfer., 4(1), 625-632, 2003.

Michallet, H. and Ivey, G. N.: Experiments on mixing due to internal solitary waves breaking on uniform slopes, Journal of Geophysical Research: Oceans., 104, 13467-13477, doi:10.1029/1999JC900037, 1999.

Miyata, M.: An internal solitary wave of large amplitude, La Mer. 23, 43-48, 1985.

Miyata, M.: Long Internal Waves of Large Amplitude. Springer, Berlin, pp. 399-406. doi:10.1007/9783-642-83331-1_44. 1988.

OpenFOAM User Guide 2019 http://www.openfoam:docs/.

OpenFOAM User Guide 2019 http://www.openfoam.com/documentation/user-guide.

Osborne, A. R. and Burch, T. L.: Internal solitons in the Andaman Sea, Science., 208, 451-460, doi:10.1126/science.208.4443.451, 1980 . 
Rayson, M. D., Ivey, G. N., Jones, N. L., Fringer, O. B.: Resolving high-frequency internal waves generated at an isolated coral atoll using an unstructured grid ocean model, Ocean Modelling., 122, 67-84, doi:10.1016/j.ocemod.2017.12.007, 2018.

Sandstrom, H. and Elliott, J. A.: Internal tide and solitons on the Scotian Shelf: A nutrient pump at work, Journal of Geophysical Research: Oceans., 89, 6415-6426, doi:10.1029/JC089iC04p06415, 1984.

Seadawy, A. R.: Exact solutions of a two-dimensional nonlinear Schrödinger equation, Applied Mathematics Letters., 25, 687-691, doi:10.1016/j.aml.2011.09.030, 2012.

Seadawy, A. R., Lu, D., and Yue, C.: Travelling wave solutions of the generalized nonlinear fifth-order $\mathrm{KdV}$ water wave equations and its stability, Journal of Taibah University for Science., 11, 623-633, doi:10.1016/j.jtusci.2016.06.002, 2017.

Shaw, P. T., Ko, D. S., and Chao, S. Y.: Internal solitary waves induced by flow over a ridge: With applications to the northern South China Sea, Journal of Geophysical Research: Oceans., 114(C2), doi:10.1029/2008JC005007, 2009.

Simmons, H. L., Hallberg, R. W., and Arbic, B. K.: Internal wave generation in a global baroclinic tide model, Deep Sea Research Part II: Topical Studies in Oceanography., 51(25-26), 3043-3068, doi:10.1016/j.dsr2.2004.09.015, 2004.

Smedstad, O. M., Hurlburt, H. E., Metzger, E. J., Rhodes, R. C., Shriver, J. F., Wallcraft, A. J., Kara, A. B.: An operational eddy resolving 1/16 global ocean nowcast/forecast system,Journal of Marine Systems.,40, 341-361, doi:10.1016/S0924-7963(03)00024-1, 2003.

Subich, C. J., Lamb, K. G., and Stastna, M.: Simulation of the Navier-Stokes equations in three dimensions with a spectral collocation method, International Journal for Numerical Methods in Fluids., 73(2), 103-129, doi:10.1002/fld.3788, 2013.

Thiem, Ø., Carr, M., Berntsen, J., Davies, P. A.: Numerical simulation of internal solitary wave-induced reverse flow and associated vortices in a shallow, two-layer fluid benthic boundary layer. Ocean dynamics., 61, 857, doi:10.1007/s10236-011-0396-5, 2011.

Turkington, B., Eydeland, A., and Wang, S.: A computational method for solitary internal waves in a continuously stratified fluid, Studies in Applied Mathematics., 85, 93-127, doi:10.1002/sapm199185293, 1991.

Vlasenko, V., Stashchuk, N., and Hutter, K.: Baroclinic Tides: Theoretical Modeling andObservational Evidence, Cambridge University Press., doi:10.5670/oceanog.2006.107, 2005.

Vlasenko, V., Stashchuk, N., Guo, C., Chen, X.: Multimodal structure of baroclinic tides in the South China Sea, Nonlinear Processes in Geophysics., 17(5), 529-543, doi:10.5194/npg-17-529-2010, 2010.

Wang, X., Zhou, J. F., Wang, Z., You, Y. X.: A numerical and experimental study of internal solitary wave loads on semi-submersible platforms, Ocean Engineering., 150, 298-308, doi:10.1016/j.oceaneng.2017.12.042, 2018.

Wei, G., Du, H., Xu, X. H., Zhang, Y. M., Qu, Z. Y., Hu, T. Q., You, Y. X.: Experimental investigation of the generation of large-amplitude internal solitary wave and its interaction with a submerged slender 
https://doi.org/10.5194/gmd-2021-102

Preprint. Discussion started: 27 May 2021

(c) Author(s) 2021. CC BY 4.0 License.

body, Science China Physics, Mechanics and Astronomy., 57, 301-310, doi:10.1007/s11433-0135196-0, 2014.

Wilcox, D. C.: Comparison of two-equation turbulence models for boundary layers with pressure gradient, AIAA journal., 31(8), 1414-1421, doi:10.2514/3.11790, 1993.

Xu, C. and Stastna, M.: Instability and cross-boundary-layer transport by shoaling internal waves over realistic slopes, Journal of Fluid Mechanics., 895, doi:10.1017/jfm.2020.389, 2020.

Yuan, G. and Xiao-Yan, T.: A coupled variable coefficient modified KdV equation arising from a twolayer fluid system, Communications in Theoretical Physics., 48, 961-970, doi:10.1088/02536102/48/6/001, 2007.

Zhang, Z., Fringer, O. B., and Ramp, S. R.: Three-dimensional, nonhydrostatic numerical simulation of nonlinear internal wave generation and propagation in the South China Sea, Journal of Geophysical Research: Oceans., 116(C5), doi:10.1029/2010JC006424, 2011.

Zhang. H. G., Gu J. B., Jia, H. Q., Gu, B.: A numerical model for internal wave propagation in continuously stratified ocean, Chinese Journal of Theoretical and Applied Mechanics., 44, 896-903, doi:10.6052/0459-1879-12-195, 2012. 